\title{
Multi-Messenger Physics With the Pierre Auger Observatory
}

\author{
Karl-Heinz Kampert ${ }^{1}$, Miguel Alejandro Mostafa ${ }^{2}$, \\ Enrique Zas $^{3}$ and The Pierre Auger Collaboration ${ }^{4 * t}$ \\ ${ }^{1}$ University of Wuppertal, Wuppertal, Germany, ${ }^{2}$ Pennsylvania State University, University Park, State College, PA, \\ United States, ${ }^{3}$ University of Santiago de Compostela, Santiago de Compostela, Spain, ${ }^{4}$ Pierre Auger Observatory, \\ Malargüe, Argentina
}

\section{OPEN ACCESS}

Edited by:

Mohamed Chabab,

Cadi Ayyad University, Morocco

Reviewed by:

Alexandre Creusot,

Paris Diderot University, France

Annarita Margiotta,

University of Bologna, Italy

Damien Dornic,

UMR7346 Centre de Physique des Particules de Marseille (CPPM),

France

*Correspondence:

The Pierre Auger Collaboration auger_spokespersons@fnal.gov

${ }^{\dagger}$ Author names and affiliations appear at the Appendix

Specialty section:

This article was submitted to

High-Energy and Astroparticle

Physics,

a section of the journal

Frontiers in Astronomy and Space

Sciences

Received: 15 January 2019

Accepted: 22 March 2019

Published: 24 April 2019

Citation:

Kampert K-H, Alejandro Mostafa M,

Zas $E$ and The Pierre Auger

Collaboration (2019) Multi-Messenger

Physics With the Pierre Auger

Observatory.

Front. Astron. Space Sci. 6:24.

doi: 10.3389/fspas.2019.00024
An overview of the multi-messenger capabilities of the Pierre Auger Observatory is presented. The techniques and performance of searching for Ultra-High Energy neutrinos, photons and neutrons are described. Some of the most relevant results are reviewed, such as stringent upper bounds that were placed to a flux of diffuse cosmogenic neutrinos and photons, bounds placed on neutrinos emitted from compact binary mergers that were detected by LIGO and Virgo during their first and second observing runs, as well as searches for high energy photons and neutrons from the Galactic center that constrain the properties of the putative Galactic PeVatron, observed by the H.E.S.S. collaboration. The observation of directional correlations between ultra-high energy cosmic rays and either high energy astrophysical neutrinos or specific source populations, weighted by their electromagnetic radiation, are also discussed. They constitute additional multi-messenger approaches aimed at identifying the sources of high energy cosmic rays.

Keywords: UHECR, high energy neutrinos, high energy photons, high energy neutrons, multi-messenger astrophysics, compact binary mergers

\section{INTRODUCTION}

With the discovery of neutrinos from SN1987A (Bionta et al., 1987; Hirata et al., 1987) arriving $4 \mathrm{~h}$ before the light detected by conventional telescopes it became clear that there was a lot to learn from examining any type of particles and radiation coming from astrophysical objects, and that neutrino detectors could give early alerts that would facilitate the observation of the evolution of such transients from the earliest stages. Besides the different wavelengths of traditional astronomy, neutrinos, cosmic rays, very high energy gamma rays, and gravitational waves provide complementary information to study the most energetic objects of the Universe. While the SN1987A event might be said to mark the onset of multi-messenger astronomy, the term was only introduced in the late 1990s (see e.g., Barwick, 2000; Halzen, 2003), and the final boost to the field took place quite recently with the emergence of both neutrino (Aartsen et al., 2014) and gravitational-wave astronomy (Abbott et al., 2016b). Indeed the discovery of gravitational waves from the merging of a neutron star binary system by LIGO and Virgo (Abbott et al., 2017a) triggered a spectacular series of observations in the full electromagnetic spectrum from radio to the very high energy gamma rays (Abbott et al., 2017c) and searches for neutrino fluxes with ANTARES, IceCube, and the Pierre Auger Observatory (Albert et al., 2017a). The combined effort marks an unprecedented leap forward in astrophysics revealing many aspects of the Gamma-Ray Burst (GRB) induced by the merger and its subsequent kilonova. More recently an energetic neutrino candidate was detected in IceCube in coincidence with the powerful blazar TXS-0506+056 during a flare in the very-high-energy gamma-ray band, incompatible with a chance 
correlation at the $3 \sigma$-level (Aartsen et al., 2018a). After scanning archival data, possible evidence for enhanced neutrino emission (quantified at $3.5 \sigma$-level) was also found from this direction, during an independent 5-month period between September 2014 and March 2015 (Aartsen et al., 2018b). It is now quite clear that there is much potential in the combined analysis of data from different experiments and multi-messenger astronomy has excellent prospects of making many more significant contributions in the near future.

The Pierre Auger Observatory is the largest and most precise detector of ultra-high energy air showers, such as those regularly induced by cosmic rays. While cosmic rays are expected to have curved trajectories in their path to Earth and thus lose time correlation with light emission, at the highest energies they could still keep directional information on the as-yet unidentified sources where they are produced. The study of the arrival directions of these particles has revealed deviations from isotropy (Aab et al., 2017d, 2018b) that, combined with other particle searches, could be of relevance for multi-messenger astronomy. Indeed spatial correlations have been searched for between the arrival directions of the highest energy cosmic rays and classes of objects that have been proposed as sources in the very high-energy regime such as Active Galactic Nuclei (AGN) and Starburst Galaxies (SBG) (Aab et al., 2018a). Notably, it has also been shown that it is possible to detect neutral particles of sufficiently high energy with the Pierre Auger Observatory. By looking at the depth development of the showers, it is relatively easy to identify neutrinos which interact in the lower layers of the atmosphere. Photons can also be discerned from the background of cosmic rays because the produced showers have a reduced number of muons and they develop deeper than cosmic rays in the atmosphere. Finally, there is no known possibility to separate neutron-induced showers from the charged cosmic rays on the basis of the shower development but, since neutrons are directional, it is in principle possible to identify sources of nearby neutrons by looking at an excess from given directions or to exploit potential time and directional correlations. This procedure could also be applied to any type of neutral particles that induce a shower in the atmosphere such as photons.

In this article, we review the capability of the Observatory to search for signals of such particles and discuss the contributions that have been made.

\section{THE PIERRE AUGER OBSERVATORY}

The Pierre Auger Observatory is designed to detect extensive air showers produced by primary cosmic rays above $0.1 \mathrm{EeV}$. It is located near the city of Malargüe, Argentina, at a latitude of $35.2^{\circ} \mathrm{S}$, a longitude of $69.2^{\circ} \mathrm{W}$ and at an approximate altitude of $1400 \mathrm{~m}$ above sea level, or equivalently an atmospheric depth $X_{\text {ground }}=880 \mathrm{~g} \mathrm{~cm}^{-2}$. It comprises a surface detector (SD) array of 1,660 water-Cherenkov stations deployed over a triangular grid of $1.5 \mathrm{~km}$ spacing and a system of 27 telescopes grouped in four sites forming the fluorescence detector (FD). The telescopes are erected at the periphery of the Observatory to observe the atmosphere over the full area of $3,000 \mathrm{~km}^{2}$ covered by the SD array. The SD stations sample the density of the secondary particles of the air shower at the ground and are sensitive to the electromagnetic, muonic and hadronic components. The FD observes the longitudinal development of the air shower by detecting the fluorescence and Cherenkov light emitted during the passage of the secondary particles of the shower in the atmosphere. Unlike the SD, the fluorescence telescopes are operated only during clear and moonless nights, for an average duty cycle of about $14 \%$. The hybrid feature of the Auger Observatory combining these two well-established techniques has proved to be extremely rewarding in making it the most precise instrument to reconstruct the energy, mass, and direction of Ultra-High Energy Cosmic Rays (UHECRs). Details about the different detector components and their performances can be found in Aab et al. (2015c) and Abraham et al. (2010).

Besides its general hybrid capabilities, a feature that makes the Pierre Auger Observatory a very versatile and powerful multimessenger observatory is the specific design of the SD stations. They are constructed as cylinders of $3.6 \mathrm{~m}$ diameter and 1.2 $\mathrm{m}$ height filled with 12 tones of purified water, each. Charged particles entering a station induce emission of Cherenkov light which is reflected at the walls by a diffusive Tyvek liner, and collected by three 9-inch photomultiplier tubes (PMT) at the top surface and in optical contact with the water. The PMT signals are sampled by flash analog-to-digital converters (FADC) with a time resolution of $25 \mathrm{~ns}$ (Abraham et al., 2010) ${ }^{1}$. This provides good discrimination of electrons and muons entering the detector station from the top, a feature which is important to identify muon-poor air showers induced by photons, not only with the FD but also with the SD. Moreover, the detector stations also provide a large cross section for inclined and up-going air showers, a feature that is of key-importance for the detection of neutrino-induced air showers. Both of these aspects will be discussed in more detail below.

The modular structure of the surface detector array and fluorescence telescopes allowed the data taking to start in 2004 in a partial configuration. In 2008 the detector was completed and since then data have been taken, practically without interruption. Once installed, the local stations work practically without external intervention. There are two types of trigger conditions: a local trigger at the level of an individual station (second order or T2 trigger), and a global trigger (third order or T3 trigger) to register events. The T2 trigger condition is satisfied when either the signal exceeds the equivalent of 3.2 Vertical Equivalent Muons (VEM) in at least one time bin of the signal trace in the FADC - the so-called "threshold trigger" - or when it exceeds a much lower threshold (0.2 VEM) in at least 13 bins within a $3 \mu$ s time window (i.e., 120 bins) - the so-called "Time-over-threshold (ToT) trigger." The ToT condition was designed to trigger on signals broad in time, characteristic of the electromagnetic component dominant over the first 1,000 g $\mathrm{cm}^{-2}$ of the extensive air shower, and it is crucial for neutrino identification as explained below. The data acquisition system receives the local T2 triggers and builds a global T3 trigger requiring a relatively compact configuration of at least three

${ }^{1}$ The recorded sequence of signals every $25 \mathrm{~ns}$ is referred to as the signal trace. 
local stations compatible in time, each satisfying the ToT trigger, or four triggered stations within a time window slightly larger than light travel time with any type of T2 trigger (Aab et al., 2015c). With the completed array, the global T3 trigger rate is about two events per minute, one third being showers with energies above $3 \times 10^{17} \mathrm{eV}$.

\section{NEUTRINOS}

Neutrinos can travel in straight paths from the confines of the Universe, are capable of going through large matter depths without absorption, and are excellent messengers from extragalactic sources, where protons or nuclei are thought to be accelerated to high energies. Neutrinos are also produced from cosmic-ray interactions with the cosmic microwave background. Besides pointing to the position of their sources, they can also provide valuable information concerning hadronic acceleration processes, composition, the local environment at the sources, and their evolution with redshift (Gaisser et al., 1995).

The idea of using inclined showers to search for neutrino interactions is old (Berezinsky and Smirnov, 1975), and the large potential of the Pierre Auger Observatory for neutrino detection was already recognized in its design stages (Capelle et al., 1998). Inclined showers induced by cosmic rays were observed in Haverah Park (Hillas et al., 1969), in the early days of extensive air shower arrays. It was at the beginning of the 2000 s when the muon patterns of the showers at ground level were sufficiently understood to allow shower reconstruction (Ave et al., 2000) (see also Aab et al., 2014e). These showers traverse large atmospheric depths, and their electromagnetic component gets almost completely absorbed before the shower particles reach ground level. As a result, for zenith angles exceeding $\sim 60^{\circ}$, the shower front reaching the SD detector stations consists mainly of muons that typically have energies between 20 and $200 \mathrm{GeV}$ and travel tens of $\mathrm{km}$ without decaying. These muons are little affected by interactions, except for continuous energy loss and deflections in the Earth's magnetic field (Ave et al., 2000). As their radius of curvature is a few thousands $\mathrm{km}$, they do not deviate much from their initial trajectories, keeping the timing of the shower front sharp.

As opposed to cosmic rays, inclined neutrinos can interact deep in the atmosphere because their interaction length exceeds the matter depth of the atmosphere for any zenith angle, $\theta$. The resulting "young" showers are rich in electrons and photons at ground level. These showers induce signals typically spread over much wider time intervals than cosmic rays with the same $\theta$ because of multiple scattering of the electrons and photons in the shower front. The broader digitized signal traces recorded at the particle detectors of the SD array resemble those produced by vertical air showers and it is thus relatively easy to single them out from the sharply arriving fronts produced by inclined protons or nuclei. Tau neutrinos interact in the Earth just below the surface and produce tau leptons that escape into the atmosphere, decaying in flight and producing up-going air showers (LetessierSelvon, 2001; Fargion, 2002). For neutrino energies exceeding 100 $\mathrm{PeV}$, the combined conversion and exit probability is maximal for nearly horizontal ("Earth-skimming") directions that exit with an elevation angle between $1^{\circ}$ and $2^{\circ}$ above the horizontal direction (Zas, 2005). These showers develop close and almost parallel to the ground. As the shower particles, particularly electrons and photons, spread laterally they can reach the SD stations, inducing characteristic signals which are also easily identified because of their broader time spread.

\subsection{Selection and Identification}

The event selection, the neutrino identification, and the exposure calculations are performed separately for Earth-skimming (ES) and down-going (DG) showers (Aab et al., 2015a). To select ES showers, all triggered events involving at least three stations are considered. The ellipsoidal signal pattern at the ground is required to have a large eccentricity (characterized by a ratio of major and minor axes greater than 5), and the direction of the major axis indicates the azimuthal angle of the event. The apparent speed of the signal at the ground, measured for each pair of stations and averaged over all of the pairs, must be in the interval $[0.967,1.034] c^{2}$, and the r.m.s. spread must be less than $0.267 c^{3}$. For the DG selection (Abreu et al., 2011a) a minimum of four triggered stations is required to reduce noise from random triggers, and two zenith angle groups are made:"Low" (DGL) and "High" (DGH), respectively, for $60^{\circ}<\theta<75^{\circ}$ and $75^{\circ}<\theta<$ $90^{\circ}$. The selection of DGL showers is just made requiring the reconstructed zenith angle to be in the $58.5^{\circ}-76.5^{\circ}$ range allowing for uncertainties. It is obtained fitting a plane to the positions of the triggered stations and the start time of the signals. For the selection of DGH showers, the reconstructed $\theta$ must exceed $75^{\circ}$, the apparent average speed of the signal at the ground must be smaller than $1.044 c^{4}$, its spread must be smaller than $0.08 c$, and the eccentricity must exceed a value of 3 .

The ES (DG) neutrino identification has been optimized using extensive shower simulations for different energies, arrival directions, decay (interaction) depths, and impact parameters, with standard software such as AIRES Sciutto (1999) and CORSIKA Heck et al. (2000) and including the SD response of the individual detector stations using specific software (Argiro et al., 2007; Aab et al., 2015c) based on GEANT4 (Agostinelli et al., 2003). A single variable has been chosen for the identification procedure in both searches. Its distribution for neutrino simulations is compared to that of regular showers obtained from a small sample of the data assumed to be exclusively cosmic rays. To select neutrino candidates, a cut is made on this variable at a value that would just yield one cosmic-ray in 50 years according to the extrapolated background distribution. For instance, in the ES case, this variable is the average area over peak $(A o P)$ value of the signal traces of each event. The area is the integrated charge of the signal trace and the peak is its maximum value, both normalized to one for vertical throughgoing muons used for on-line calibration. The cut obtained is $A o P>1.83$, ensuring sufficient time spread in the signal to reject cosmic-ray showers (Aab et al., 2015a).

\footnotetext{
${ }^{2}$ Actual values used are $[0.29,0.31] \mathrm{m} \mathrm{ns}^{-1}$.

${ }^{3} 0.08 \mathrm{~m} \mathrm{~ns}^{-1}$.

${ }^{4} 0.313 \mathrm{~m} \mathrm{~ns}^{-1}$
} 
Events with just three stations are further required to have AoP exceeding 1.4. For DG showers, a Fisher discriminant approach combines several variables based on the $A o P$ of a few selected stations. For the DGH case, the multivariate Fisher method (Fisher, 1936) uses nine variables obtained with the AoP values of the four earliest stations and a tenth variable accounting for the asymmetry in the time distribution of the earliest and latest stations of the event. For the DGL case, the Fisher variable combines five or six variables obtained from the AoP of the four or five stations closest to the shower core and their product. For DGL showers, $75 \%$ of the triggers are also required to be of special “ToT" kind (Aab et al., 2015a). For optimization purposes of DG neutrinos, further subdivisions have been made using the number of triggered stations for the DGH set and the reconstructed $\theta$ for the DGL set. The condition for a neutrino candidate is slightly different in each subset.

\subsection{Efficiency and Aperture}

The effective aperture of the detector (aperture from now on) is defined so that when it is multiplied by the neutrino spectral flux, it gives the energy distribution of the detection rate. For point sources in the DG case, this aperture is simply calculated integrating the interaction and detection probabilities over the SD area. Three separate apertures are calculated according to interaction type and neutrino flavor: (1) neutral current for all flavors and charged current muon neutrinos, where the shower is produced by the nuclear debris (typically carrying about $20 \%$ of the neutrino energy); (2) charged current for electron neutrinos where all the energy is converted to the air shower (80\% induced by the electron and the rest by nuclear fragments); (3) charged current for tau neutrinos where the shower can be produced either by the nuclear debris ( $20 \%$ of the energy) or, deeper in the atmosphere, by the tau decay ( $80 \%$ of the energy). The three apertures can be added under the assumption of equal fluxes for each neutrino flavor, as expected because of neutrino flavor oscillations in the characteristic long baselines provided by extragalactic distances to plausible sources (Learned and Pakvasa, 1995; Athar et al., 2000).

On the other hand, only tau neutrinos can be efficiently detected in the ES mode. When muon neutrinos convert to high energy muons that exit the Earth, practically all of them also exit the atmosphere without decaying because of their longer lifetime and when electron neutrinos convert to electrons the shower develops rapidly in the Earth before exiting. In the ES case, the aperture is calculated as an integral over the Earth's area (transverse to the flux direction), weighted by the probability of a tau neutrino interacting and producing a tau which subsequently exits the Earth, decays in the atmosphere and induces a shower that triggers the SD and is effectively selected and identified. This is done in two steps. The differential probability of a tau lepton of given energy exiting the Earth has been calculated as a function of $\theta$ using simulations of tau neutrino interactions in rock that include regeneration (Abreu et al., 2013). The tau-exit probability must be integrated over decay distance weighted by the survival probability, the decay probability per unit distance and the probability of detection with the SD. The detection probability for both DG and ES showers includes trigger, selection and identification and it is also obtained from the simulations.

The exit and detection probabilities in the aperture calculation depend strongly on $\theta$. A given source located at right ascension $\alpha$, and declination $\delta$ in equatorial coordinates, is observed with a zenith angle that oscillates with time according to:

$$
\cos \theta(t)=\sin \lambda \sin \delta+\cos \lambda \cos \delta \sin (2 \pi t / T-\alpha) .
$$

Here $\lambda=-35.2^{\circ}$ is the latitude of the detector, $t$ is the sidereal time, and $T$ the duration of a sidereal day. Naturally, the aperture is strongly dependent on source position and time. At any given instant the field of view of the Observatory for each of the ES, DGH and DGL channels is limited to the bands corresponding to the zenith angle range of the channel, as displayed in Figures 2 (left), 3 for specific examples discussed below.

Typically, the search for neutrinos from point sources is performed on a pre-selected time interval chosen to match plausible emission times according to theoretical models describing the mechanisms acting within the sources. The expected event rate at the detector is calculated by integrating the aperture over the neutrino flux and, if no candidate events are found in a given time interval, a 90\% C.L. upper limit to the flux normalization is obtained matching the expected number of detected events over the time interval to 2.39 (Feldman and Cousins, 1998). An assumption about the energy spectrum of the flux has to be made. It is customary to again assume a canonical $E_{v}^{-2}$. In this case, $90 \%$ of the detected events at the Observatory are between $100 \mathrm{PeV}$ and $25 \mathrm{EeV}$ (Aab et al., 2015a). The most effective channel for neutrino detection is ES because the target mass provided by the Earth is large compared to the atmosphere and also because the threshold energy is low. For the ES channel algorithms have been found to identify events with just three triggered stations. It has been shown that Earth-skimming events can only be observed for zenith angles between $90^{\circ}$ and $95^{\circ}$, corresponding to declination angles $-55^{\circ}<\delta<60^{\circ}$. The exposure is maximal for $\delta \simeq-53^{\circ}$ and $\delta \simeq 55^{\circ}$. The apertures provided by the DGH and DGL are progressively smaller because, as the zenith angle decreases, the atmosphere offers less target mass in which the neutrinos can interact and be identified. As the search is effectively performed only for $\theta$ between $60^{\circ}$ and $95^{\circ}$, the field of view of the Observatory for neutrinos is limited to equatorial declinations between $\sim-85^{\circ}$ and $\sim 60^{\circ}$.

When the pre-selected time interval is short, much less than a day, the aperture can be very different depending on the source position and the observation time. The instantaneous aperture reaches its maximum if the source position is just below the horizon with $\theta$ between $90^{\circ}$ and $95^{\circ}$. While the source is in the ES field of view, the instantaneous aperture for neutrinos of energies above $100 \mathrm{PeV}$ exceeds that of other neutrino telescopes. Also, depending on declination, the source can be inside the ES field of view for different lengths of time. For $\delta=0^{\circ}$ there are two transit periods per day into the ES field of view of about $25 \mathrm{~min}$ each. For the optimal declination positions $\left(\delta \simeq-53^{\circ}\right.$ and $\left.\delta \simeq 55^{\circ}\right)$, near the extremes in declination of the ES band, the total transit time per day is more than $4 \mathrm{~h}$, as can be seen in Figures 2 (left), 3 . 

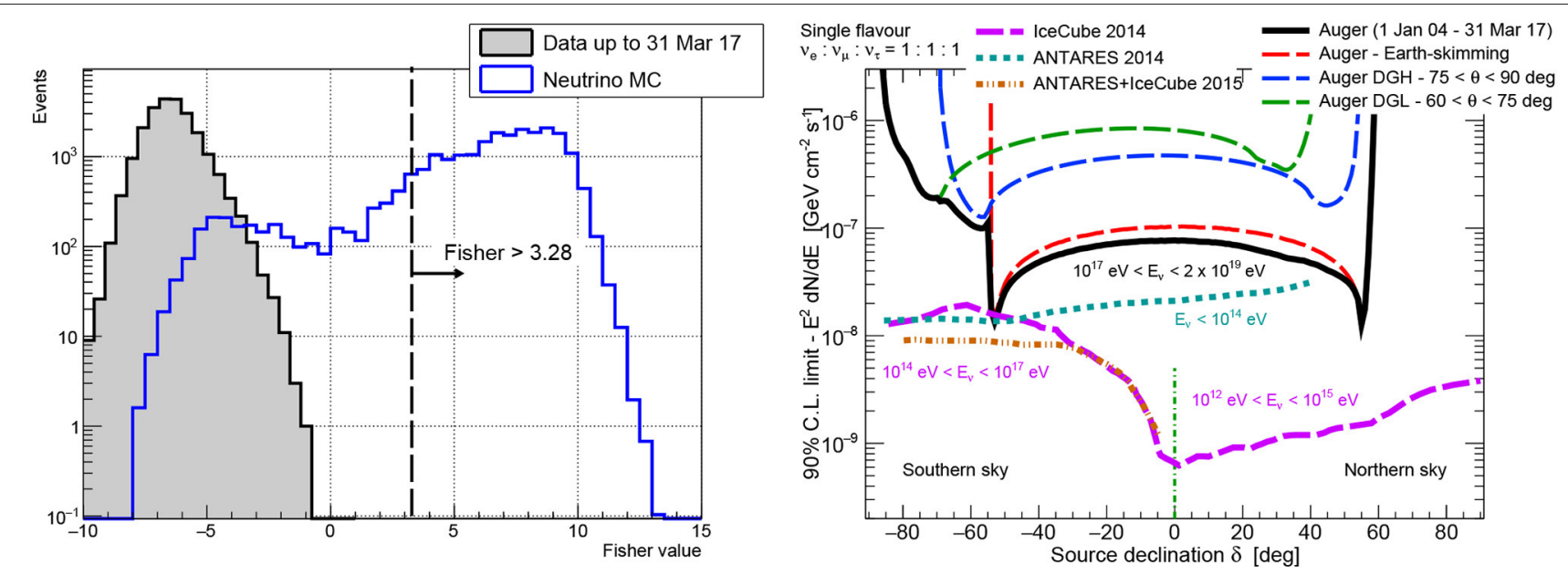

FIGURE 1 | (Left) Distribution of the Fisher variable for the DGH search (see text) for simulations compared to a small fraction of the data and assumed to be due to cosmic rays. The cut is made at a value of the Fisher variable of 3.28. (Right) Limits to the point source fluxes as a function of equatorial declination obtained from the non-observation of ES and DGH neutrino candidates up to March 31st 2017 (from Zas, 2018).

The selection and identification procedures have been applied to the data registered by the Observatory. As no neutrino candidates have been found, upper limits to the ultra-high energy (UHE) neutrino fluxes have been obtained. Upper limits to the diffuse flux with important implications for some models of UHECR production were first obtained in the ES channel (Abraham et al., 2008, 2010) and subsequently extended to include the DGH (Abreu et al., 2011b) and the DGL (Aab et al., 2015a) channels. Upper limits to point source fluxes were obtained for the ES and DGH channels (Abreu et al., 2012). Upper limits on neutrino fluxes from point sources are shown in Figure 1 (right) as a function of declination for the search period up to 31 March 2017. Articles with further updates and details on both diffuse and point source searches are to appear soon.

\subsection{Correlated Searches of Neutrinos}

The detection of gravitational waves from the merging of binary systems has marked the birth of gravitational wave astronomy. The first signals from the merging of two Black Hole (BH) systems GW150914 (Abbott et al., 2016b) and GW151226 (Abbott et al., 2016a) were reported in 2016. The luminosity distances of the two events were deduced to be $410_{-180}^{+160}$ and $440_{-190}^{+180} \mathrm{Mpc}$ and the radiated energies $3.0_{-0.5}^{+0.5}$ and $1.0_{-0.2}^{+0.1}$ solar masses, respectively. They triggered the search for the emission of electromagnetic radiation and neutrinos (Aab et al., 2016d; Adrian-Martinez et al., 2016), even though the black hole systems are not expected to emit any other type of radiation unless matter debris and magnetic fields can be found in their neighborhood, possibly remaining from the $\mathrm{BH}$ progenitors (Kotera and Silk, 2016; Murase et al., 2016).

Neutrinos were searched for with the Pierre Auger Observatory in two periods of time: $\pm 500 \mathrm{~s}$ around the UTC times at which the mergers were observed and 1 day following their occurrence (Aab et al., 2016d). These intervals were motivated by the association of the merging of compact objects to Gamma-Ray Bursts (GRBs) (Mészáros, 2006; Moharana et al., 2016; Perna et al., 2016). Neutrinos have been postulated to be produced by accelerated cosmic rays interacting with the GRB photons in the prompt phase and with the lower energy photons of the afterglow. The 1,000 s time window is an upper bound of the duration of the prompt GRB phase (Albert et al., 2017b; Kimura et al., 2017) while the 1-day window is a conservative bound of the duration of the afterglow (Mészáros, 2006).

No neutrino candidates were observed in coincidence with these events in either of the time windows. Assuming a flux $k^{G W} E_{v}^{-2}$, where $k^{G W}$ is a normalization constant, $90 \%$ C.L. limits on the neutrino emission in the $\mathrm{EeV}$ from these events were reported as fluence limits. The spectral fluence is defined as $E_{v}^{2} \phi\left(E_{v}\right) \Delta t$. Here $\phi$ is the spectral flux and $\Delta t$ is the chosen time interval over which the emission is assumed to take place and during which it is also assumed to be constant. In these conditions the spectral fluence is related to the total energy emitted in neutrinos. For the assumed spectrum it is trivial to convert the spectral fluence to an upper limit for the total energy emitted in neutrinos in a given energy band, which can be compared to the energy radiated in gravitational waves. The reported limits were obtained for a 1-day period. Due to the poor localization of these events the upper limits, shown in Figure 2 (left) for GW150914, are reported as a function of declination. A similar limit was obtained for GW151226 since the distances to both mergers are quite similar. The most restrictive limit assuming emission during a single day for GW150914 $(\mathrm{GW151226})$ is obtained for $\delta=-53^{\circ}\left(\delta=55^{\circ}\right)$ and would correspond to a total energy radiated in UHE neutrinos smaller than $7.7 \times 10^{53}\left(9.7 \times 10^{53}\right)$ erg when integrated from $100 \mathrm{PeV}$ to $25 \mathrm{EeV}$. This corresponds to $14.3 \%$ (44.1\%) of the radiated energy in gravitational waves. The limits found by IceCube and ANTARES apply to lower neutrino energies and give more restrictive limits on the total energy radiated in these neutrinos (Adrian-Martinez et al., 2016). 


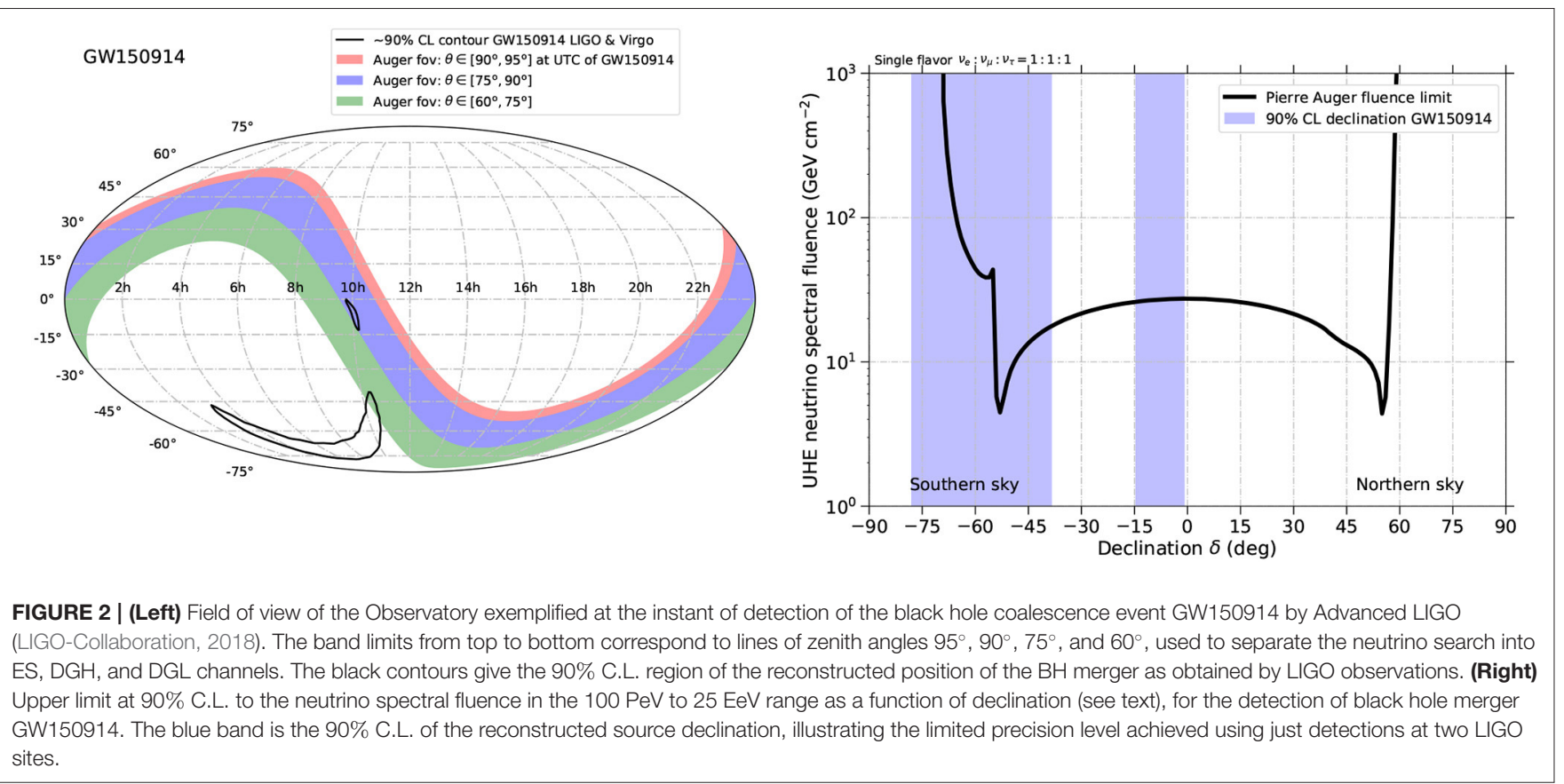

Models have been proposed where black hole mergers accelerate UHECR (Kotera and Silk, 2016), and the energy budget of these collapsing events could account for all of the UHECR with a modest fraction of the gravitational wave energy (of order 3\%) going to UHECR acceleration. Assuming that a similar fraction goes into UHE neutrinos, about 0.5 events could have been expected at the Observatory in coincidence with GW150914 in the most optimistic scenario. The upper limits were obtained averaging the instantaneous aperture over a day. If the emission time was shorter than 1 day, more stringent limits would be obtained provided the source was in the neutrino field of view during the neutrino emission, especially if it was in the ES field of view. Due to the poor localization of both events, it is not possible to know if the events were in the neutrino field of view at the detection time. Given the $90 \%$ C.L. contour of GW151226 (GW150917) the overlap with the field of view of the neutrino search is $68.9 \%$ (13\%) for a time window of $1,000 \mathrm{~s}$. The overlap over a whole sidereal day is $100 \%$ in both cases. The detection of black hole mergers closer to us and with more directional precision could provide more stringent constraints for such models.

The observation of a neutron star merger GW170817 by the LIGO-Virgo collaboration (Abbott et al., 2017b) less than two seconds before the short GRB 170817A detected by FermiLAT (Goldstein et al., 2017; Savchenko et al., 2017) from a coincident direction, has had outstanding implications. The successful alert systems triggered observations and detection in practically all bands of the electromagnetic spectrum in an unprecedented way, marking the onset of a new era of Multimessenger Astronomy (Abbott et al., 2017c). Early optical observations allowed a precise localization slightly off-centered in the galaxy NGC 4993 at equatorial coordinates $\alpha(J 2000.0)=$ $13^{\mathrm{h}} 09^{\mathrm{m}} 48^{\mathrm{s}}, \delta(J 2000.0)=-23^{\circ} 22^{\prime} 53$. $^{\prime \prime} 343$ (Coulter et al., 2017).

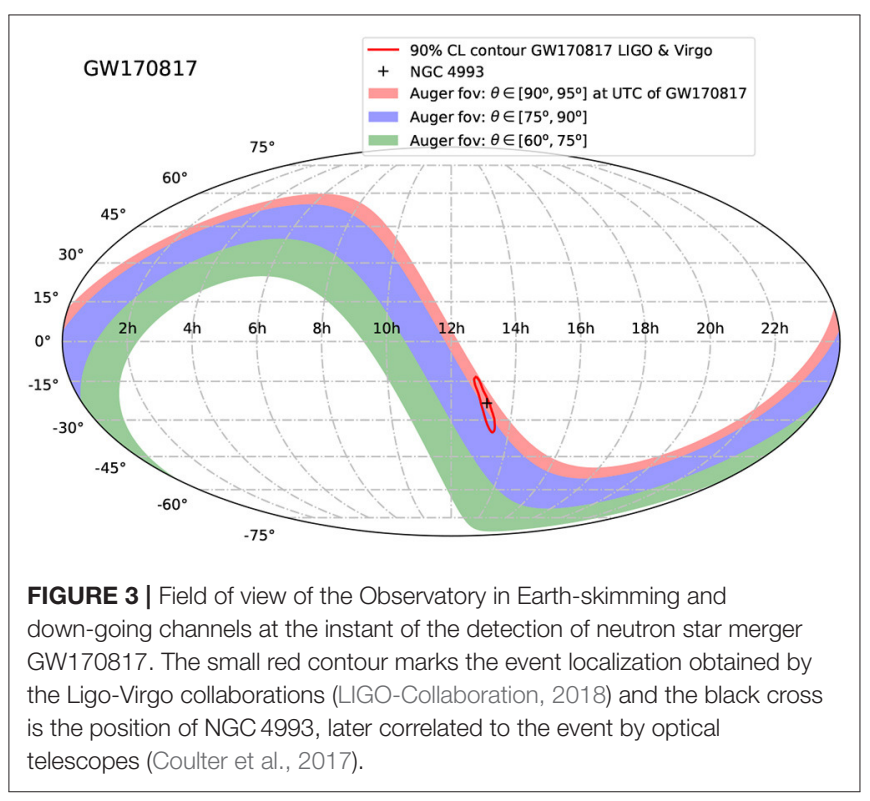

Searches for neutrino events were also performed at neutrino telescopes and at the Pierre Auger Observatory (Albert et al., 2017a). At the time of the GW detection, the source was located at a zenith angle of $91.9^{\circ}$ at the Observatory site, just below the horizon and extremely close to the sweet-spot for Earthskimming neutrinos, see Figure 3. When considered in a time interval of $\pm 500 \mathrm{~s}$ about the detection $\left(93.3^{\circ}<\theta<90.4^{\circ}\right)$, the $\mathrm{EeV}$ exposure is larger than that of dedicated neutrino telescopes and provides the most stringent upper limit to the neutrino fluence at 90\% C.L. in the $100 \mathrm{PeV}$ to $25 \mathrm{EeV}$ interval (Albert et al., 2017a), complementary to IceCube and ANTARES, as 


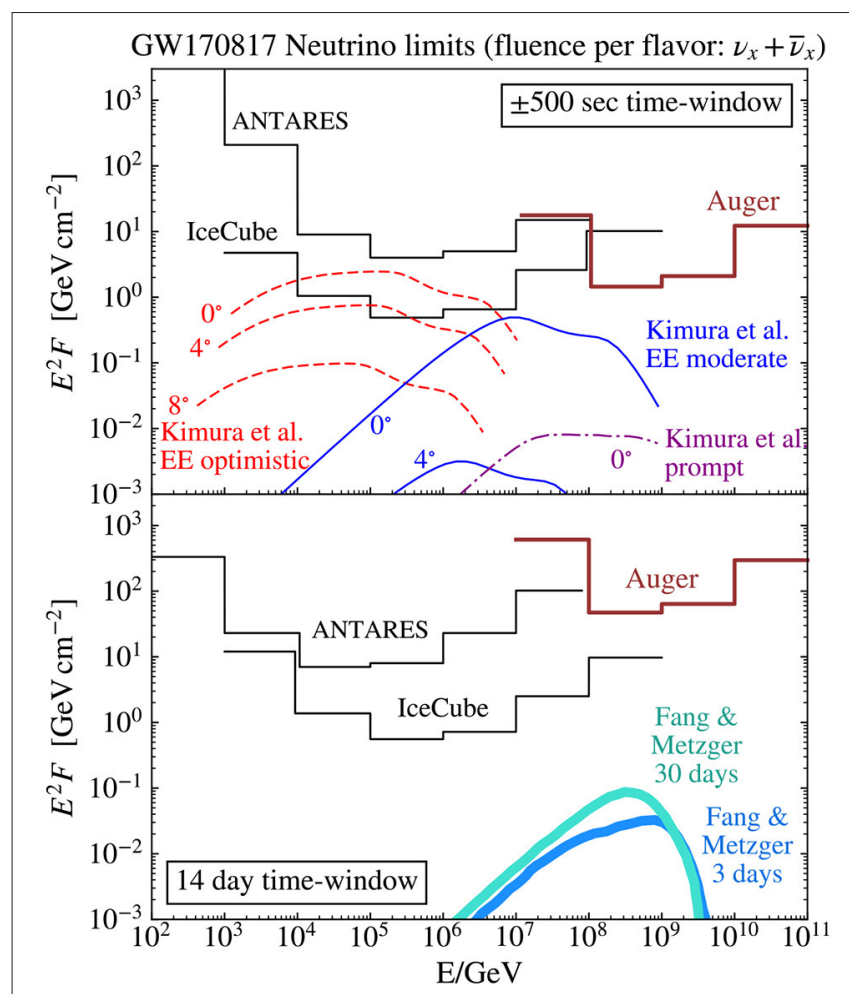

FIGURE 4 | Upper limits at 90\% C.L. on the neutrino spectral fluence from GW170817 for a 1,000 s period centered at (14 day after) the time of the event in the top (bottom) panel. The bounds in the top panel are compared to predictions of models of prompt and extended emission (EE) (Kimura et al., 2017 ) in the case of exact alignment of the line of sight to the rotation axis and for selected off-axis viewing angles. The bounds obtained for a 14-day period on the bottom panel are compared to models of longer lived emission (Fang and Metzger, 2017). All models have been scaled to $40 \mathrm{Mpc}$, the distance to the host galaxy NGC 4993 (Modified from Albert et al., 2017a).

illustrated in Figure 4. The analysis was also made for a longer time window of 14 days matching predictions from longer-lived emission processes (Gao et al., 2013; Metzger, 2017) and also displayed in Figure 4. Neutrino bounds obtained with the Pierre Auger Observatory and other neutrino telescopes are within the range predicted in some models of GRB from neutron star mergers. IceCube bounds allowed constraints to be placed on the orientation angle relative to the jet axis (assumed to be coincident to the rotation axis) for one of the most optimistic models.

Acceleration of hadrons in astrophysical objects inevitably leads to photon and neutrino production with similar energy fluences. On September 17th of 2017, a $290 \mathrm{TeV}$ neutrino was detected at the IceCube telescope (Aartsen et al., 2017) in Antarctica and subsequent correlated gamma-ray observations pointed for the first time to a powerful blazar in a flaring state, TXS 0506+056, as a candidate source of the neutrino (Aartsen et al., 2018a). This result was further supported by a neutrino burst from the same direction of the sky obtained in a time window of about 158 days when searching for time correlations from the same direction (Aartsen et al., 2018b). A search for possible coincident signals at the Auger Observatory was also performed when the results were made public. This source is $21 \%$ of the sidereal day in the field of view of the neutrino search but it was not in the field of view at the exact time of the neutrino detection. No neutrinos were found (Alvarez Muñiz, 2018). The details of this search and the bounds obtained are to be reported in a separate article.

\section{PHOTONS}

\subsection{Photon Identification}

Ultra-high energy (UHE) photons are among the possible particles contributing to the flux of cosmic rays and may arise from a number of processes. Firstly, UHE photons are expected from the GZK-process just like UHE neutrinos and can be used to probe the parameter space of UHECR sources. Secondly, they may be produced by hadronic interactions of cosmic rays within the sources or within their local environment. In these cases, the photons are produced on average with around $10 \%$ of the energy of the primary incident proton. Thirdly, a large flux of UHE photons has been predicted in top-down models with UHECR originating from the decay of supermassive particles such as topological defects or Super Heavy Dark Matter (SHDM) particles (see e.g., Bhattacharjee and Sigl, 2000; Aloisio et al., 2015).

As opposed to neutrinos, photons undergo interactions with the extragalactic background light (EBL) inducing electromagnetic cascades. This process makes photons sensitive to the extragalactic environment (e.g., EBL and magnetic fields) but it also limits the volume from which EeV photons may be detected. It is small compared to the GZK-sphere, but large enough to encompass the Milky Way, the Local Group of galaxies, and possibly Centaurus A, given an attenuation length of about 4.5 Mpc at EeV energies (De Angelis et al., 2013). The secondary electrons and positrons created by pair production in the photon fields can again interact with background photons via inverse-Compton scattering, resulting in an electromagnetic cascade that ends at $\mathrm{GeV}-\mathrm{TeV}$ energies. Comparing these expected diffuse $\mathrm{GeV}-\mathrm{TeV}$ fluxes measured with instruments sensitive in this energy range provides another important cross-link to $\gamma$-astronomy (see e.g., Alves Batista et al., 2016).

The search for UHE photon primaries is based on the different development and particle content of electromagnetic and hadronic air showers. The induced electromagnetic cascades develop slower than hadronic ones so that the shower maximum $X_{\max }$ is reached closer to the ground. Proton and photon simulated showers have average $X_{\max }$ values that differ by about $200 \mathrm{~g} \mathrm{~cm}^{-2}$ in the EeV energy range. This difference is enhanced at energies above $10 \mathrm{EeV}$ because of the LandauPomeranchuk-Migdal (LPM) effect (Landau and Pomeranchuk, 1953; Migdal, 1956). Above $50 \mathrm{EeV}$, photons also have a non-negligible probability to convert in the geomagnetic field (Erber, 1966; McBreen and Lambert, 1981; Homola et al., 2007) producing a bunch of low-energy electromagnetic particles, called "pre-shower", entering the atmosphere. The $X_{\max }$ of the pre-showered cascades is smaller than for non-converted ones and the separation between the average $X_{\max }$ for photons and proton correspondingly reduced. The fluorescence detector (FD) with its high resolution of about $15 \mathrm{~g} \mathrm{~cm}^{-2}$ and systematic 
uncertainties of less than about $8 \mathrm{~g} \mathrm{~cm}^{-2}$ for $X_{\max }$ (Aab et al., 2014c; Bellido, 2018) is an ideal instrument to discriminate photon- from hadron-induced air showers with high sensitivity even in single events. Thus the FD is able to provide strong constraints on the photon fraction of UHECR.

It is also possible to search for photon showers using the SD because the shower development and nature of the primary cosmic ray affect the content and the shape of the distribution of particles at the ground as a function of the distance from the shower axis (Lateral Distribution Function, LDF). Photoninduced showers generally are expected to have a steeper LDF compared to hadron primaries because of the sub-dominant role played by the flatter muonic component. The high-energy effects (LPM and pre-showering) affect the average muon content of air showers by typically less than $20-50 \%$ dependent on zenith angle, which is small compared to the factor 5-8 difference in muons with regards to hadronic showers (Homola et al., 2007; Risse and Homola, 2007). However, the different stage of shower development at the ground (relative to $X_{\max }$ ) leads to a modification of the observed LDF. Given the steeper LDF and the muon-driven SD triggers, the footprint at the ground, and consequently the number of triggered stations, $N_{\text {stat }}$, is typically smaller for electromagnetic showers (Abreu et al., 2011b). These features can be combined into a single observable $S_{b}$, that discriminates the photon- and hadron-induced air showers (Aab et al., 2016b). Discrimination on a shower by shower basis is less efficient compared to the FD, but given the much larger statistics of the $\mathrm{SD}$, strong constraints on the photon fluxes can be provided. For hybrid observations, the discriminating observables $X_{\max }, S_{b}$, and $N_{\text {stat }}$ are injected into a Boosted Decision Tree (BDT) algorithm that takes into account also the energy and angular dependencies of the discriminating observables. To identify photons, a cut is defined at the median of the BDT response distribution for simulated photons, as depicted in Figure 5 (left). We note that the discrepancy between the data and the proton simulations is in agreement with the current experimental indications of a composition varying from light to heavier in the EeV range (Aab et al., 2014d) and with the muon deficit observed in simulations with respect to the Auger data (Aab et al., 2015b, 2016b). Applying the cut in this conservative way, the signal efficiency remains constant independently of the composition and hadronic model assumptions. Events having a BDT response larger than the median cut are selected as "photon candidates". This yields a background contamination of 0.14 \% for proton showers using QGSJET-II-04. This background level overestimates the one expected in data because the composition is generally heavier than that of pure protons and because the interaction models underestimate the muon number in EAS, making the showers look more photon-like. If the multivariate analysis is performed with a mixture of $50 \%$ proton and $50 \%$ iron as input to the training phase, the background contamination reduces to $\sim 0.04 \%$ with the main contribution coming from the smaller values of the simulated $X_{\max }$. At present, such probabilities are to be considered systematic uncertainty to the background of potential photon candidates, i.e., such background events would dilute the photon limits.

\subsection{Photon Searches}

Figure 5 (right) shows, as a result, the upper limits on the integral photon flux derived from 9 years (Jan. 2005-Dec. 2013) of hybrid and SD data compared to results from other experiments (Aab et al., 2017e). Eight candidates were found in the first two energy intervals. These events are close to the applied photon cut and detailed simulations of hadronic showers will need to be performed to judge the probability that they are caused by UHECR rather than by photons. For now, they are conservatively considered background rather than signal so that the upper limits of the first two energy bins become less stringent. The differential flux limit between 10 and $30 \mathrm{EeV}$ is found to be 6.80 . $10^{-11} \mathrm{GeV} \mathrm{cm}^{-2} \mathrm{~s}^{-1} \mathrm{sr}^{-1}$ at $90 \%$ C.L. Some top-down scenarios proposed to explain the origin of trans-GZK cosmic rays (dashed lines) are illustrated and rejected by previous bounds on the photon flux. A recent super-heavy dark matter proposal (SHDM II) developed in the context of an inflationary theory is shown as a long-dashed line (Aloisio et al., 2015). Constraints on the lifetime-and-mass parameter space of SHDM particle will be imposed by current and future limits on the photon flux, as obtained for example in Kalashev and Kuznetsov (2016).

A prime interest in the search for photons is to identify the first UHE photon point sources or-in case of non-observationsto provide relevant upper limits thereby constraining the source characteristics. As already mentioned, the horizon is limited to only a few Mpc which reaches out to Cen A but not much farther (Aab et al., 2017a). If the energy spectra of $\mathrm{TeV} \gamma$ sources measured by atmospheric Cherenkov telescopes (Hinton and Hofmann, 2009) extend to $\mathrm{EeV}$ energies, it is plausible that photons and neutrons from these sources are detectable also by the Auger Observatory. Sources that produce particle fluxes according to an $E^{-2}$ energy spectrum inject equal energy into each decade. Thus, a measured energy flux of $1 \mathrm{eV} \mathrm{cm}^{-2} \mathrm{~s}^{-1}$ in the $\mathrm{TeV}$ decade, as is found for a number of Galactic sources, would result in the same energy flux in the $\mathrm{EeV}$ decade if the spectrum continues to such high energies and energy losses during propagation are negligible. A source of specific interest is the galactic center for which the H.E.S.S.-collaboration measured a gamma-ray flux up to about $50 \mathrm{TeV}$ without any observation of a cutoff or a spectral break, suggesting that the Galactic center hosts a peta-electron volt accelerator, called "PeVatron" (Abramowski et al., 2016).

It is still debated whether these photons are produced in hadronic processes. An interesting test for this is provided by a search for neutrons from this direction, because neutrons would necessarily be produced in charge exchange interactions of protons at the source. This will be discussed in section 5 . The ratio between photon and neutron emissivities from $\mathrm{p}-\mathrm{p}$ collisions at the same primary proton energy depends primarily on the spectral index of the protons at the source. Assuming a continuation of the parent proton spectrum with a spectral index $\Gamma_{p} \lesssim 2.4$ well beyond the energies observed in the photon spectrum, the photon emissivities are expected to dominate over those of neutrons (Crocker et al., 2005).

To search for photons from a list of predefined target sources (Aab et al., 2017a), more relaxed cuts are applied to the observed 

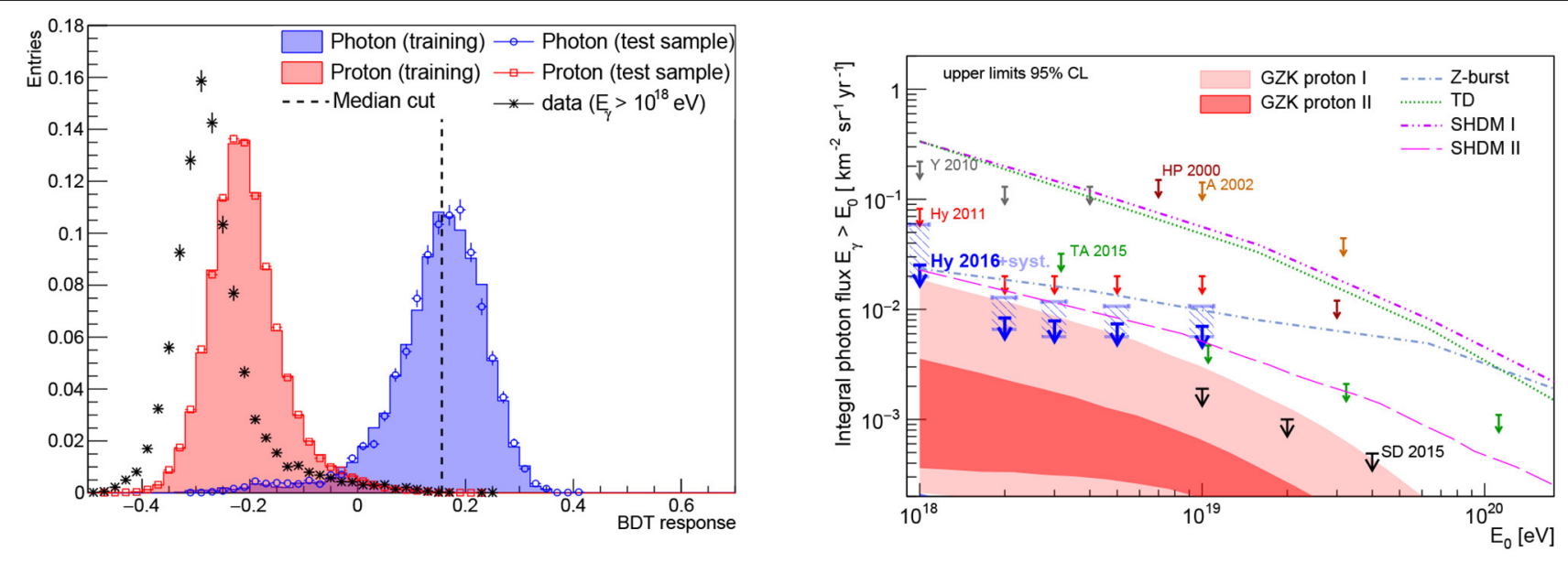

FIGURE 5 | (Left) Photon identification with a Boosted Decision Tree for signal (photon, blue), background (proton, red) and data (black). For simulations, both the training and the test samples are shown. The cut at the median of the photon distribution is indicated by the dashed line. QGSJET-II-04 is used as high-energy hadronic interaction model. (Right) Compilation of upper limits on the integral photon fluxes from Aab et al. (2017e). Blue arrows: Integral photon upper limits from the 9 year hybrid data sample assuming a photon flux following $E^{-2}$ and with no background subtraction. The limits obtained when the detector systematic uncertainties are taken into account are shown as horizontal segments (light blue) delimiting a dashed-filled box at each energy threshold; Black arrows: Nine year SD data sample (Bleve, 2016). Previous data from Auger as well as data from TA, AGASA, Yakutsk, and Haverah Park are included for comparison. The lines and shaded regions give the predictions for top-down models and GZK photon fluxes, respectively, assuming different parameters (references can be found in Aab et al., 2017e).

events (Aab et al., 2014a). For each candidate source direction an optimized cut in the multivariate output distribution is determined which depends on the expected number of isotropic background events in that direction. This number is calculated by applying a scrambling technique that takes into account detector efficiencies and aperture features. For each target direction, a top-hat counting region of $1^{\circ}$ is assumed (Aab et al., 2014a). Averaging over all considered target directions, the multivariate cut is expected to retain $81.4 \%$ of photons while rejecting $95.2 \%$ of background hadrons. In none of the sources and source classes could $\mathrm{EeV}$ photons be detected. As an example, the result for the Galactic center is illustrated in Figure 6. Conservatively, the extrapolation of the H.E.S.S. data to $\mathrm{EeV}$ energies does not take into account the increase of the p-p cross-section by about $40 \%$ relative to $\mathrm{TeV}$ energies. The current upper limit of 0.044 $\mathrm{eV} \mathrm{cm}^{-2} \mathrm{~s}^{-1}$ (95\% C.L., $\Gamma=2$ ) at energies above $0.2 \mathrm{EeV}$ can already constrain the allowed parameter space for a flux continuation to EeV energies.

The observation of photon fluxes from individual sources or from stacked sets of targets would have proved that $\mathrm{EeV}$ protons are being accelerated at those considered sources within the galaxy or its neighborhood. However, the null results leave open the possibility that protons observed at EeV energies are of extragalactic origin. This is in fact suggested by the large scale anisotropies observed with the Auger Observatory (Aab et al., 2017d). Moreover, the absence of detectable photon fluxes, as reported here, does not exclude the production of $\mathrm{EeV}$ protons within the Galaxy because the derived flux limits are timeaveraged values. EeV photons might be produced in transient sources, such as gamma-ray bursts or supernovae, or be aligned in jets not pointing to us. Extending the searches to bursting sources of EHE photons is a goal of ongoing multi-messenger analyses within Auger, briefly addressed in section 7 .

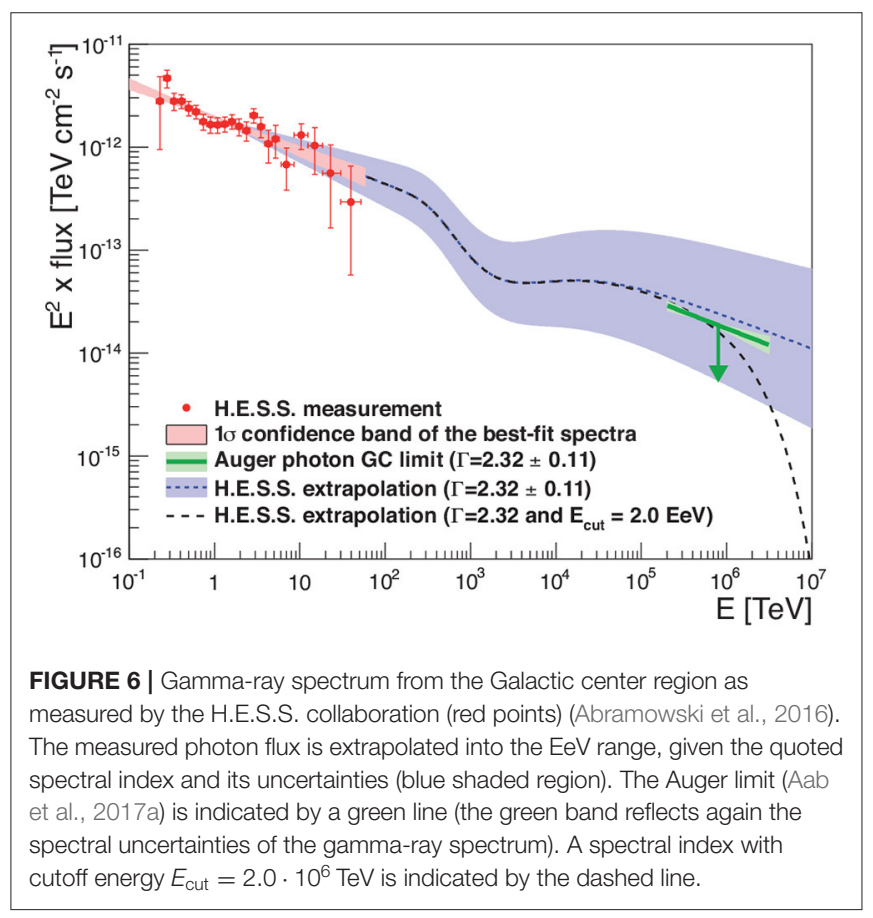

\section{NEUTRONS}

Like photons and neutrinos, neutrons travel in straight lines, undeflected by magnetic fields. They produce air showers that are indistinguishable from those produced by protons. Thus, a flux of neutrons from a discrete source would cause an excess of cosmic-ray events around the direction to the source, clustered within the angular resolution of the Observatory. Since free 
neutrons undergo $\beta$-decay with a mean lifetime of about $880 \mathrm{~s}$ at rest, the mean travel distance for relativistic neutrons is 9.2 $\mathrm{kpc} \times E_{\mathrm{n}} / \mathrm{EeV}$. The distance from Earth to the Galactic center is about $8.3 \mathrm{kpc}$, and the radius of the Galaxy is approximately $15 \mathrm{kpc}$. Thus, sources in part of the Galactic disk, including the Galactic center, should be detectable via neutrons above $1 \mathrm{EeV}$. Above $2 \mathrm{EeV}$, the volume for detectable neutron emitters includes most of the Galaxy.

Neutron production is governed by charge exchange interactions of high energy cosmic-ray protons with ambient photons, protons, or nuclei leading to the creation of a $\pi^{+}$. meson. The $\pi^{+}$takes the positive charge of the proton and a leading neutron emerges with most of the energy that the proton had. The production of neutrons via creation of $\pi^{+}$-mesons is necessarily accompanied by photons originating from decay of similarly produced neutral pions. However, photons resulting from the decay of neutral pions acquire only a small fraction of the proton energy, so that the production of neutrons exceeds the hadronic production of photons of the same energy, provided the accelerated proton spectrum falls approximately like $1 / E^{2}$ or more steeply with energy. This makes searches of high energy neutrons a highly relevant and sensitive probe for Galactic hadronic cosmic ray accelerators.

Similarly to the targeted search of $\mathrm{EeV}$ photon sources, discussed in section 4, a flux of neutrons from astrophysical sources in the Galaxy can be detected in the Pierre Auger Observatory as an excess of cosmic-ray air showers arriving from the direction of the sources. To avoid the statistical penalty for making many trials, classes of objects were tested in combinations as "target sets". Those target sets include msec pulsars (PSR), pulsar wind nebulae (PWN), microquasars, and magnetars (the full list of targets can be found in Aab et al., 2014b). In addition, a search for a neutron flux from the Galactic center and from the Galactic plane was performed. Within a target set, each candidate source is weighted in proportion to its electromagnetic flux, its exposure to the Auger Observatory, and its flux attenuation factor due to neutron decay.

None of the searches provided evidence for a neutron flux from any class of candidate sources. Based on the first 9 years of data (Jan. 1, 2004-Oct. 31, 2013), the upper limits on the energy flux from these candidate sources, including the Galactic center, are mostly at a value of $0.10-0.15 \mathrm{eV} \mathrm{cm}^{-2} \mathrm{~s}^{-1}$ (Aab et al., 2014 b), which is about a factor of 10 below the energy fluxes detected from $\mathrm{TeV}$ gamma-ray sources in the Galaxy (Hinton and Hofmann, 2009) and at about the level of sensitivity reached with the targeted photon searches, discussed above. If those sources were accelerating protons in the same environment to $\mathrm{EeV}$ energies with the $1 / E^{2}$ dependence expected for Fermi acceleration, then the energy flux of neutrons in the EeV energy decade would even exceed the energy flux in $\mathrm{TeV}$ gamma rays.

Similarly, the energy flux of neutrons from the Galactic plane could be derived to less than $0.56 \mathrm{eV} \mathrm{cm}^{-2} \mathrm{~s}^{-1}$ (95\% C.L.). This provides a stringent constraint on models for continuous production of $\mathrm{EeV}$ protons in the Galaxy because the injection rate of protons into the Galactic disk must be sufficiently strong to balance their escape from the Galaxy. The concomitant neutron emission rate is model dependent. It could exceed the proton emission rate if protons are magnetically bound to the sources and only the produced neutrons escape, yielding $\mathrm{EeV}$ protons by their later decays. More likely, however, the neutron luminosity at any fixed energy is less than the proton luminosity. Based on an estimate of the proton emission rate, the limits on the neutron flux from the Galactic plane could be used to derive an upper limit on the ratio $\eta=$ neutron luminosity/proton luminosity. It was found to be $\eta_{\mathrm{UL}} \simeq 0.006$ (Aab et al., 2014b), which is a significant constraint on models for continuous production of EeV protons in the Galaxy.

\section{DIRECTIONAL CORRELATIONS OF UHECRS WITH NEUTRINOS AND SOURCE CANDIDATES}

Even the most energetic cosmic rays can be subject to significant deflection and corresponding energy-dependent time delays. The strength and properties of the extragalactic magnetic field (EGMF) causing these effects remain largely unknown, and UHECR observed from bursting and continuous sources have already helped to provide constraints to the EGMF (see e.g., Lemoine et al., 1997; Bray and Scaife, 2018; Mollerach and Roulet, 2018). In section 3.3 we have discussed the time-correlated observation of neutrinos from merging binary systems. In fact, black hole mergers are also expected to produce UHECR and may provide sufficient luminosity to power the UHECR acceleration up to the highest energies (Kotera and Silk, 2016). On the other hand, $100 \mathrm{EeV}$ protons from an extragalactic bursting source located at a distance of $30 \mathrm{Mpc}$ are expected to arrive with time delays relative to photons and neutrinos by $\mathcal{O}(1-$ 1,000) years (Lemoine et al., 1997) and may even reach out to $10^{6} \mathrm{yrs}$ in extreme cases, so that UHECR could not be included in those combined directional and temporal searches in a straightforward way.

\subsection{Search for UHECR-Neutrino Correlations}

Purely directional correlations between high-energy neutrinos and UHECR still provide an interesting target of opportunity because UHECRs accelerated in astrophysical sources are naturally expected to produce high-energy photons and neutrinos in interactions with the ambient matter and radiation. If neutrinos result from the decays of pions produced in $p \gamma$ or $p p$ processes, they would carry about 3-5\% of the proton energy. Hence, neutrinos observed with energies of $30 \mathrm{TeV}$ to $2 \mathrm{PeV}$, such as observed by IceCube, would have been produced by protons with energies in the $1-100 \mathrm{PeV}$ range. These energies are much smaller than those registered by the Auger Observatory, and it is possible that the sources that produce the $\mathrm{PeV}$ neutrinos do not accelerate CRs up to ultra-high energies. In that case no correlations would be expected. On the other hand, the sources that produce the UHECR can also be expected to produce lower energy CRs. If the observed neutrinos come from the same sources as the UHECR, some degree of correlation in the arrival directions of the highest energy cosmic rays and the observed neutrinos could be expected depending on composition and 
on the strength of magnetic fields. It should also be noted that neutrinos can reach us from cosmological distances while the UHECR horizon is limited to only a few $100 \mathrm{Mpc}$. Thus, only a small fraction of the detected neutrinos could be expected to have originated from visible UHECR sources so that correlations, if they were found, cannot be very prominent.

After some initial cross-correlation efforts by the ANTARES collaboration (Adrián-Martínez et al., 2013; Aublin et al., 2018) different analysis strategies have been devised in an intercollaborative effort of IceCube and Auger (Aartsen et al., 2015) that were more recently joined also by the ANTARES and Telescope Array (TA) collaborations (Aartsen et al., 2016; Al Samarai et al., 2018).

In a classical cross-correlation method, the number of observed UHECR-neutrino pairs is counted as a function of the maximal angular separation and then compared to the averaged background, which is calculated once for an isotropic neutrino flux and once for an isotropic UHECR flux. Applying this analysis to the latest combined Auger+TA UHECR and IceCube neutrino data sets, it was found that a maximum departure from the expectation for an isotropic CR flux, keeping the arrival directions of the neutrinos fixed, occurs at an angular distance of $1^{\circ}$ for tracks and $22^{\circ}$ for cascades, with post-trial $p$-values of 0.48 and $5.4 \cdot 10^{-3}$, respectively (Al Samarai et al., 2018). In parallel, an unbinned likelihood method was used. It is based on a stacking point-source analysis applied to the UHECRs, where the neutrino arrival directions are smeared with a symmetric 2D-Gaussian and stacked as the signal template. The width of the Gaussian is the quadratic sum of the UHECR reconstruction uncertainty and the magnitude of the magnetic deflection, which is assumed to be described by $\sigma_{\mathrm{MD}}=6^{\circ} / E_{\mathrm{CR}}[100 \mathrm{EeV}]$. Again, the analysis is applied to track- and cascade-like high-energy neutrino candidates separately. Assuming an isotropic flux of neutrinos, the smallest post-trial $p$-values are found to be $2.2 \cdot 10^{-2}$ and $1.7 \cdot 10^{-2}$ for the track- and cascade-like events, respectively.

These results are interesting, but the level of correlation is still too small to arrive at a conclusion. It will be interesting to see how the signal evolves as more data are accumulated by the neutrino and UHECR observatories involved. In addition, new improved analysis strategies are being developed making, for example, better use of the measured UHECR composition and energy so that deflections in the EGMF and Galactic magnetic field can be more appropriately accounted for.

\subsection{Association of UHECR With Source Populations}

A related multi-messenger approach aimed at identifying the sources of UHECR from their directional information is to search for correlations with catalog based astronomical objects. The basic concept is related to the studies presented in the previous section, only that the tracers are specific source populations rather than samples of high energy neutrinos. A set of source candidates that was studied in Aab et al. (2018a) includes nearby radio-loud AGNs and SBGs ${ }^{5}$, selected as possible sources to accelerate CRs to the highest energies (Hillas, 1984; Dermer and Razzaque, 2010). Individual objects found within a given catalog are expected to contribute differently to the total UHECR flux observed at Earth. Since the CR luminosity of individual sources is not known, we have chosen their observed electromagnetic emission as a proxy. In case of the 17 radio loud $\gamma \mathrm{AGN}$ found within $250 \mathrm{Mpc}$ in the 2FHL catalog from Fermi-LAT (Ackermann et al., 2016), the measured integral $\gamma$-flux from $50 \mathrm{GeV}$ to $2 \mathrm{TeV}$ was used as a proxy for the UHECR flux. Similarly, 23 (out of a total of 63) SBGs within $250 \mathrm{Mpc}$ and with a flux larger than $0.3 \mathrm{Jy}$ were taken from (Ackermann et al., 2012) and weighted with their continuum radio emission at $1.4 \mathrm{GHz}$. In both cases, attenuation of UHECRs from distant objects in the photon background fields was accounted for using a data-driven scenario that reproduces the composition and spectral constraints obtained by the Observatory (Aab et al., 2017b). Within this Ansatz, the relative contributions of NGC 4945, NGC 253, and M83 are expected to be about 38, 33, 13\% of the total UHECR flux from SBGs observed at the Auger Observatory. The relative contributions seen at the Observatory from the $\gamma$ AGN would be dominated by the Cen A Core (75\%), followed by M87 (15\%) and Mkn 421 (8\%).

To test these models against the observed UHECR sky maps, a maximum-likelihood analysis was performed with two free parameters aimed at maximizing the degree of correlations of the model maps with UHECR events: (a) the fraction of an isotropic component contributing to the total UHECR flux in addition to the source population being tested, and (b) the width of a $2 \mathrm{D}$ Gaussian smearing around the position of the source candidates. The first free parameter can be interpreted as the contribution from more distant or less luminous sources, and the second parameter effectively accounts for the random scattering of UHECR in the EGMF. This analysis is repeated for a sequence of energy thresholds applied to the UHECR events and the test statistic (TS) as a function of the two parameters is analyzed. The results are shown in Figure 7. It was found that the SBG-Model rejects the hypothesis of an isotropic sky best at $E_{\mathrm{CR}}>39 \mathrm{EeV}$ and yields a posttrial significance of $4.0 \sigma$. Here, the SBGs contribute about $10 \%$ to the total flux, and the smearing angle is $12.9^{\circ}$. In the case of the $\gamma \mathrm{AGN}$ model, the best rejection of isotropy is reached at $E_{\mathrm{CR}}>60 \mathrm{EeV}$ at a level of $2.7 \sigma$ with a $\gamma \mathrm{AGN}$ fraction to the total flux of about $7 \%$ and a smearing angle $6.9^{\circ}$. Interestingly, the smearing angle decreases with increasing energy as expected from the larger rigidities of the UHECR in the $\gamma$ AGN model.

The results are very promising and demonstrate departures from a pure isotropic sky with more and more structure becoming visible, starting at above $8 \mathrm{EeV}$ with the observation of a large-scale dipole (Aab et al., 2017d), indications of higher order moments above $16 \mathrm{EeV}$ (Aab et al., 2018b), blurry spots above $40 \mathrm{EeV}$, and hints of sharper spots above $60 \mathrm{EeV}$. There

\footnotetext{
${ }^{5}$ We note that the potential of SGBs to accelerate particles to the highest energies
} has been questioned, recently (Romero et al., 2018; Matthews et al., 2019). 

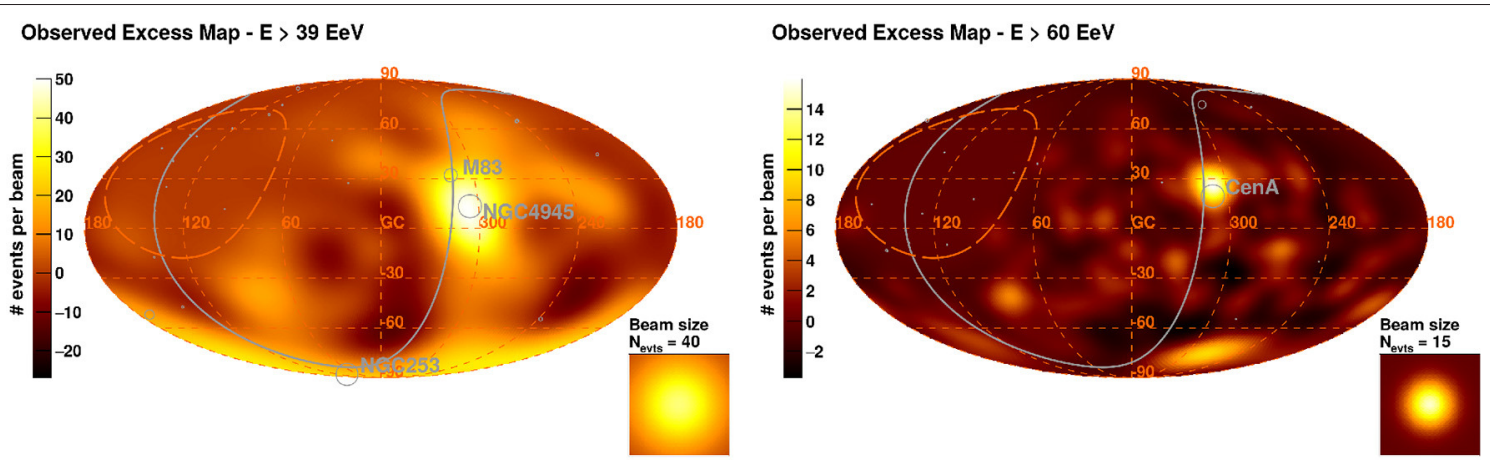

Model Excess Map - Starburst galaxies - E > $39 \mathrm{EeV}$
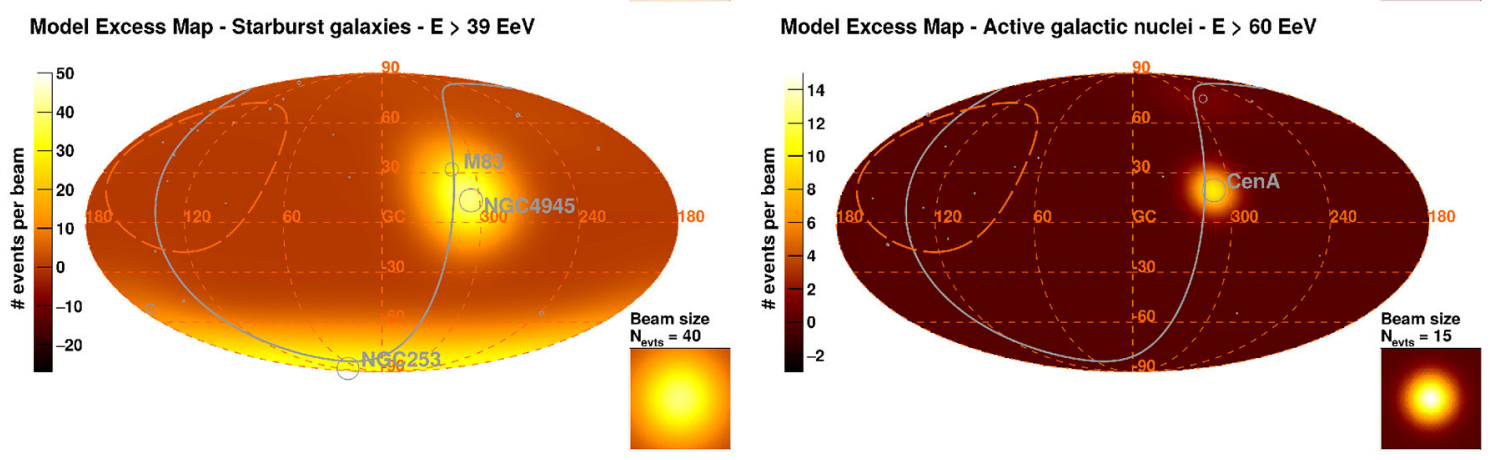

FIGURE 7 | (Top row) observed excess maps for $E_{\mathrm{CR}}>39 \mathrm{EeV}$ and $E_{\mathrm{CR}}>60 \mathrm{EeV}$. (Bottom row) model excess maps for the SGB (left) and $\gamma$ AGN model (right) at the same energy thresholds as above. The color scales indicate the number of events per smearing beam (see inset). The model flux map corresponds to a uniform full-sky exposure. The supergalactic plane is shown as a solid gray line. An orange dashed line delimits the field of view of the array (from Aab et al., $2018 a$ ).

are indications that both SGB and AGN, as well as a mixture of both or similar populations could play a significant role in the production of UHECR. However, it is still too early to conclude about this long standing problem. Future analyses, possibly profiting from full-sky observations with TA, may involve a better modeling of the Galactic magnetic field, account for the change of the composition as a function of energy, and possibly introduce better proxies for the unknown UHECR luminosities, so that $5 \sigma$ detections are well within reach.

\section{CONCLUSIONS AND PROSPECTS}

The Pierre Auger Observatory was designed as a multi-purpose observatory for the high-energy Universe with multi-messenger observations being foreseen from the beginning. In this review, we have summarized a few prominent examples that demonstrate the unprecedented sensitivities to UHE photons, neutrinos, and neutrons. Observations of photons in the so far dark Universe at $E_{\gamma} \gtrsim 10^{17} \mathrm{eV}$ or of a neutrino at these energies would be a major breakthrough by itself. However, the non-observation of point sources and diffuse fluxes of photons and neutrinos allowed the derivation of upper bounds that constrain models very effectively. The bounds to diffuse photon and neutrino fluxes have ruled out the top-down models of UHECRs origin motivated by particle physics and also started to constrain the GZK-effect as a dominant process for explaining the observed flux suppression of the most energetic UHECR (Zas, 2018).
Many TeV $\gamma$-sources are observed at energy fluxes of the order of $1 \mathrm{eV} \mathrm{cm}^{-2} \mathrm{~s}^{-1}$. Such sources would be visible to the Auger Observatory as strong photon and Galactic neutron sources if their energy spectrum would continue with a Fermi-like energy distribution up to about $10^{17} \mathrm{eV}$. Again, their absence suggests that their maximum source energy does not reach out to the threshold energy of the Observatory and/or that their spectrum is significantly softer than $d J / d E \propto E^{-2}$.

Point source searches now routinely include also mergers of compact binaries alerted by gravitational wave interferometers. The most spectacular event so far was the neutron star merger GW170817 at a distance of about $40 \mathrm{Mpc}$. Within the predefined $\pm 500 \mathrm{~s}$ search window, the Auger Observatory reached a neutrino flux sensitivity above $100 \mathrm{PeV}$ that was over an order of magnitude higher than of any other neutrino observatory presently operated. Again, the absence of neutrinos at Auger, IceCube and ANTARES allowed constraining the jet properties of the neutron star merger. The third observation run $\mathrm{O} 3$ is about to start with many more events being expected in the near future. To accommodate for this, mechanisms are set up to automatically react to GW or other astrophysical alerts and search for neutrinos and photons.

While receiving alerts from a worldwide network of observatories, it is also possible to send alerts from the Observatory. The Auger Observatory is both a triggering and a follow-up partner in the Astrophysical Multi-messenger Observatory Network (AMON) (Smith et al., 2013), which establishes and distributes alerts for immediate follow-up 
by subscribed observatories with private or Gamma-Ray Coordinates Network ${ }^{6}$ notices. AMON registers all vertical $(\theta \leq$ $\left.60^{\circ}\right)$, high-quality, Auger SD events, with energy above $3 \mathrm{EeV}$ with a cadence of at most a few minutes. AMON establishes clusters of two or more Auger events received correlated in time and arrival direction as alerts. These are excesses of events expected from neutral (photon or neutron) UHECRs as discussed in sections 4 and 5 . Unlike the aforementioned neutron and photon searches, this alert channel is sensitive to ultra-high energy neutral transient emission which is less well constrained in the time-integrated searches. For each Auger alert, AMON also receives and transmits the background rate, in other words, the significance of each of the alerts, which depends on the energy and declination of each event. Thus high-significance alerts can be preferentially followed-up. To improve the overall significance of alerts and to enhance future photon and neutrino searches in general, additional improved hardware triggers have been implemented to the existing surface detector array of the Observatory. Besides enhancing the sensitivity to photons and neutrinos, they allow reducing their detection thresholds. Thus, photon alerts for individual ultra-high energy photon candidates from a selection of events which utilizes observables based on the new triggers will be transmitted in near-real time and available for immediate follow-up using the AMON infrastructure. Finally, the infilled array, covering an area of $23.5 \mathrm{~km}^{2}$ with a $750 \mathrm{~m}$ detector spacing, will provide acceptance for neutrinos and photons below the present detection threshold, though with reduced total exposure. It may also be used to generate specific alerts and there are plans to extend the neutrino search to the FD (Aramo et al., 2005).

Presently, the Pierre Auger Observatory is being upgraded to AugerPrime primarily to improve the mass composition measurements and particle physics capabilities with the surface detector array (Aab et al., 2016c; Engel, 2016; Martello, 2017). For this purpose, $3.8 \mathrm{~m}^{2}$ plastic scintillation detectors will be installed on each of the existing surface detector stations and the current readout electronics will be replaced by a faster and more powerful one. The new electronics will facilitate three times faster sampling of the signals $(120 \mathrm{MHz}$ instead of $40 \mathrm{MHz})$, will have more monitoring features implemented, and will have more sensitive triggers installed for low-energy showers and those initiated by photons and neutrinos. Moreover, the infilled array will be furnished with large area underground muon detectors (Aab et al., 2016a, 2017c) and each surface detector station will be amended by a radio antenna to provide improved shower information for inclined air showers (Hörandel, 2018). AugerPrime will be operated into 2025 and will improve the statistics for composition information and composition enhanced astronomy by about a factor of 10. Clearly, all of these features will open up many new possibilities for improved searches of photons and neutrinos. This suit of enhancements will further strengthen the prominent role of the Pierre Auger Observatory as a multi-messenger observatory for the next decade.

${ }^{6} \mathrm{https} / /$ gcn.gsfc.nasa.gov.

\section{AUTHOR CONTRIBUTIONS}

All authors listed have made a substantial, direct and intellectual contribution to the work, and approved it for publication.

\section{ACKNOWLEDGMENTS}

The successful installation, commissioning, and operation of the Pierre Auger Observatory would not have been possible without the strong commitment and effort from the technical and administrative staff in Malargüe. We are very grateful to the following agencies and organizations for financial support:

Argentina - Comisión Nacional de Energía Atómica; Agencia Nacional de Promoción Científica y Tecnológica (ANPCyT); Consejo Nacional de Investigaciones Científicas y Técnicas (CONICET); Gobierno de la Provincia de Mendoza; Municipalidad de Malargüe; NDM Holdings and Valle Las Leñas; in gratitude for their continuing cooperation over land access; Australia - the Australian Research Council; Brazil - Conselho Nacional de Desenvolvimento Científico e Tecnológico (CNPq); Financiadora de Estudos e Projetos (FINEP); Fundação de Amparo à Pesquisa do Estado de Rio de Janeiro (FAPERJ); São Paulo Research Foundation (FAPESP) Grants No. 2010/07359-6 and No. 1999/05404-3; Ministério da Ciência, Tecnologia, Inovações e Comunicações (MCTIC); Czech Republic Grant No. MSMT CR LTT18004, LO1305, LM2015038 and CZ.02.1.01/0.0/0.0/16_013/0001402; France - Centre de Calcul IN2P3/CNRS; Centre National de la Recherche Scientifique (CNRS); Conseil Régional Ile-de-France; Département Physique Nucléaire et Corpusculaire (PNC-IN2P3/CNRS); Département Sciences de l'Univers (SDU-INSU/CNRS); Institut Lagrange de Paris (ILP) Grant No. LABEX ANR-10-LABX-63 within the Investissements d'Avenir Programme Grant No. ANR-11IDEX-0004-02; Germany - Bundesministerium für Bildung und Forschung (BMBF); Deutsche Forschungsgemeinschaft (DFG); Finanzministerium Baden-Württemberg; Helmholtz Alliance for Astroparticle Physics (HAP); Helmholtz-Gemeinschaft Deutscher Forschungszentren (HGF); Ministerium für Innovation, Wissenschaft und Forschung des Landes NordrheinWestfalen; Ministerium für Wissenschaft, Forschung und Kunst des Landes Baden-Württemberg; Italy - Istituto Nazionale di Fisica Nucleare (INFN); Istituto Nazionale di Astrofisica (INAF); Ministero dell'Istruzione, dell'Universitá e della Ricerca (MIUR); CETEMPS Center of Excellence; Ministero degli Affari Esteri (MAE); México - Consejo Nacional de Ciencia y Tecnología (CONACYT) No. 167733; Universidad Nacional Autónoma de México (UNAM); PAPIIT DGAPA-UNAM; The Netherlands - Ministry of Education, Culture and Science; Netherlands Organisation for Scientific Research (NWO); Dutch national e-infrastructure with the support of SURF Cooperative; Poland - National Centre for Research and Development, Grant No. ERA-NET-ASPERA/02/11; National Science Centre, Grants No. 2013/08/M/ST9/00322, No. 2016/23/B/ST9/01635 and No. HARMONIA 5-2013/10/M/ST9/00062, UMO2016/22/M/ST9/00198; Portugal - Portuguese national funds and FEDER funds within Programa Operacional Factores de 
Competitividade through Fundação para a Ciência e a Tecnologia (COMPETE); Romania - Romanian Ministry of Research and Innovation CNCS/CCCDI-UESFISCDI, projects PN-III-P1-1.2PCCDI-2017-0839/19PCCDI/2018, PN-III-P2-2.1-PED-20161922, PN-III-P2-2.1-PED-2016-1659 and PN18090102 within PNCDI III; Slovenia - Slovenian Research Agency; Spain Ministerio de Economía, Industria y Competitividad (FPA201785114-P and FPA2017-85197-P), Xunta de Galicia (ED431C 2017/07), - Junta de Andalucía (SOMM17/6104/UGR), Feder Funds, RENATA Red Nacional Temática de Astropartículas

\section{REFERENCES}

Aab, A., Abreu, P., Aglietta, M., Ahlers, M., Ahn, E. J., Al Samarai, I., et al. (2014a). A search for point sources of EeV photons. Astrophys. J. 789:160. doi: 10.1088/0004-637X/789/2/160

Aab, A., Abreu, P., Aglietta, M., Ahlers, M., Ahn, E. J., Al Samarai, I., et al. (2014b). A targeted search for point sources of EeV neutrons. Astrophys. J. 789:L34. doi: 10.1088/2041-8205/789/2/L34

Aab, A., Abreu, P., Aglietta, M., Ahlers, M., Ahn, E. J., Al Samarai, I., et al. (2014c). Depth of maximum of air-shower profiles at the Pierre Auger Observatory. I. Measurements at energies above $10^{17.8} \mathrm{eV}$. Phys. Rev. D 90:122005. doi: 10.1103/PhysRevD.90.122005

Aab, A., Abreu, P., Aglietta, M., Ahlers, M., Ahn, E. J., Al Samarai, I., et al. (2014d). Depth of maximum of air-shower profiles at the Pierre Auger Observatory. II. Composition implications. Phys. Rev. D 90:122006. doi: 10.1103/PhysRevD.90.122006

Aab, A., Abreu, P., Aglietta, M., Ahlers, M., Ahn, E. J., Al Samarai, I., et al. (2014e). Reconstruction of inclined air showers detected with the Pierre Auger Observatory. JCAP 1408:019. doi: 10.1088/1475-7516/2014/08/019

Aab, A., Abreu, P., Aglietta, M., Ahlers, M., Ahn, E. J., Al Samarai, I., et al. (2015a). Improved limit to the diffuse flux of ultrahigh energy neutrinos from the Pierre Auger Observatory. Phys. Rev. D 91:092008. doi: 10.1103/PhysRevD.91. 092008

Aab, A., Abreu, P., Aglietta, M., Ahlers, M., Ahn, E. J., Al Samarai, I., et al. (2015b). Muons in air showers at the Pierre Auger Observatory: mean number in highly inclined events. Phys. Rev. D 91:032003. doi: 10.1103/PhysRevD.91.032003

Aab, A., Abreu, P., Aglietta, M., Ahn, E. J., Al Samarai, I., Albert, J. N., et al. (2015c). The pierre auger cosmic ray observatory. Nucl. Instrum. Meth. A 798, 172-213. doi: 10.1016/j.nima.2015.06.058

Aab, A., Abreu, P., Aglietta, M., Ahn, E. J., Al Samarai, I., Albuquerque, I. F. M., et al. (2016a). Prototype muon detectors for the AMIGA component of the Pierre Auger Observatory. JINST 11:P02012. doi: 10.1088/1748-0221/11/02/P02012

Aab, A., Abreu, P., Aglietta, M., Ahn, E. J., Al Samarai, I., Albuquerque, I. F. M., et al. (2016b). Testing hadronic interactions at ultrahigh energies with air showers measured by the pierre auger observatory. Phys. Rev. Lett. 117:192001. doi: 10.1103/PhysRevLett.117.192001

Aab, A., Abreu, P., Aglietta, M., Ahn, E. J., Al Samarai, I., Albuquerque, I. F. M., et al. (2016c). The pierre auger observatory upgrade - preliminary design report. arXiv:1604.03637.

Aab, A., Abreu, P., Aglietta, M., Ahn, E. J., Al Samarai, I., Albuquerque, I. F. M., et al. (2016d). Ultrahigh-energy neutrino follow-up of gravitational wave events GW150914 and GW151226 with the pierre auger observatory. Phys. Rev. D 94:122007. doi: 10.1103/PhysRevD.94.122007

Aab, A., Abreu, P., Aglietta, M., Al Samarai, I., Albuquerque, I. F. M., Allekotte, I., et al. (2017a). A targeted search for point sources of $\mathrm{EeV}$ photons with the Pierre Auger Observatory. Astrophys. J. 837:L25. doi: 10.3847/2041-8213/aa61a5

Aab, A., Abreu, P., Aglietta, M., Al Samarai, I., Albuquerque, I. F. M., Allekotte, I., et al. (2017b). Combined fit of spectrum and composition data as measured by the Pierre Auger Observatory. JCAP 1704:038. doi: 10.1088/1475-7516/2017/04/038

Aab, A., Abreu, P., Aglietta, M., Al Samarai, I., Albuquerque, I. F. M., Allekotte, I., et al. (2017c). Muon counting using silicon photomultipliers
(FPA2015-68783-REDT) and > María de Maeztu Unit of Excellence (MDM-2016-0692); USA - Department of Energy, Contracts No. DE-AC02-07CH11359, No. DEFR02-04ER41300, No. DE-FG02-99ER41107 and No. DESC0011689; National Science Foundation, Grant No. 0450696; The Grainger Foundation; Marie CurieIRSES/EPLANET; European Particle Physics Latin American Network; European Union 7th Framework Program, Grant No. PIRSES-2009-GA-246806; and UNESCO. in the AMIGA detector of the Pierre Auger observatory. JINST 12:P03002. doi: 10.1088/1748-0221/12/03/P03002

Aab, A., Abreu, P., Aglietta, M., Al Samarai, I., Albuquerque, I. F. M., Allekotte, I., et al. (2017d). Observation of a large-scale anisotropy in the arrival directions of cosmic rays above $8 \times 10^{18} \mathrm{eV}$. Science 357, 1266-1270. doi: 10.1126/science.aan4338

Aab, A., Abreu, P., Aglietta, M., Al Samarai, I., Albuquerque, I. F. M., Allekotte, I., et al. (2017e). Search for photons with energies above $10^{18} \mathrm{eV}$ using the hybrid detector of the Pierre Auger Observatory. JCAP 1704:009. doi: 10.1088/1475-7516/2017/04/009

Aab, A., Abreu, P., Aglietta, M., Albuquerque, I. F. M., Allekotte, I., Almela, A., et al. (2018a). An Indication of anisotropy in arrival directions of ultra-high-energy cosmic rays through comparison to the flux pattern of extragalactic gamma-ray sources. Astrophys. J. 853:L29. doi: 10.3847/2041-8213/aaa66d

Aab, A., Abreu, P., Aglietta, M., Albuquerque, I. F. M., Allekotte, I., Almela, A., et al. (2018b). Large-scale cosmic-ray anisotropies above $4 \mathrm{EeV}$ measured by the Pierre Auger Observatory. Astrophys. J. 868:4. doi: 10.3847/1538-4357/aae689

Aartsen, M. G., Abraham, K., Ackermann, M., Adams, J., Aguilar, J. A., Ahlers, M., et al. (2015). The IceCube neutrino observatory, the Pierre Auger observatory and the telescope array: joint contribution to the 34th international cosmic ray conference (The Hague: ICRC 2015). arXiv:1511.02109.

Aartsen, M. G., Abraham, K., Ackermann, M., Adams, J., Aguilar, J. A., Ahlers, M., et al. (2016). Search for correlations between the arrival directions of IceCube neutrino events and ultrahigh-energy cosmic rays detected by the Pierre Auger Observatory and the Telescope Array. JCAP 1601:037. doi: 10.1088/1475-7516/2016/01/037

Aartsen, M. G., Ackermann, M., Adams, J., Aguilar, J. A., Ahlers, M. Ahrens, M., et al. (2014). Observation of high-energy astrophysical neutrinos in three years of IceCube data. Phys. Rev. Lett. 113:101101. doi: 10.1103/PhysRevLett.113.101101

Aartsen, M. G., Ackermann, M., Adams, J., Aguilar, J. A., Ahlers, M., Ahrens, M., et al. (2017). The IceCube neutrino observatory: instrumentation and online systems. JINST 12:P03012. doi: 10.1088/1748-0221/12/03/P03012

Aartsen, M. G., Ackermann, M., Adams, J., Aguilar, J. A., Ahlers, M., Ahrens, M., et al. (2018a). Multimessenger observations of a flaring blazar coincident with high-energy neutrino IceCube-170922A. Science 361:eaat1378. doi: $10.1126 /$ science.aat 1378

Aartsen, M. G., Ackermann, M., Adams, J., Aguilar, J. A., Ahlers, M., Ahrens, M., et al. (2018b). Neutrino emission from the direction of the blazar TXS $0506+056$ prior to the IceCube-170922A alert. Science 361, 147-151. doi: 10.1126/science.aat 2890

Abbott, B. P., Abbott, R., Abbott, T. D., Abernathy, M. R., Acernese, F., Ackley, K., et al. (2016a). GW151226: observation of gravitational waves from a 22-solar-mass binary black hole coalescence. Phys. Rev. Lett. 116:241103. doi: 10.1103/PhysRevLett.116.241103

Abbott, B. P., Abbott, R., Abbott, T. D., Abernathy, M. R., Acernese, F., Ackley, K., et al. (2016b). Observation of gravitational waves froma binary black hole merger. Phys. Rev. Lett. 116:061102. doi: 10.1103/PhysRevLett.116.061102

Abbott, B. P., Abbott, R., Abbott, T. D., Acernese, F., Ackley, K., Adams, C., et al. (2017a). GW170817: observation of gravitational waves from a binary neutron star inspiral. Phys. Rev. Lett. 119:161101. doi: 10.1103/PhysRevLett.119.161101

Abbott, B. P., Abbott, R., Abbott, T. D., Acernese, F., Ackley, K., Adams, C., et al. (2017b). Gravitational waves and gamma-rays from a binary 
neutron star merger: GW170817 and GRB 170817A. Astrophys. J. 848:L13. doi: 10.3847/2041-8213/aa920c

Abbott, B. P., Abbott, R., Abbott, T. D., Acernese, F., Ackley, K., Adams, C., et al. (2017c). Multi-messenger observations of a binary neutron star merger. Astrophys. J. 848:L12. doi: 10.3847/2041-8213/aa91c9

Abraham, J., Abreu, P., Aglietta, M., Aguirre, C., Ahn, E. J., Allard, D., et al. (2008). Upper limit on the diffuse flux of UHE tau neutrinos from the Pierre Auger Observatory. Phys. Rev. Lett. 100:211101. doi: 10.1103/PhysRevLett.100.211101

Abraham, J., Abreu, P., Aglietta, M., Aguirre, C., Ahn, E. J., Allard, D., et al. (2010). The fluorescence detector of the Pierre Auger observatory. Nucl. Instrum. Meth. A 620, 227-251. doi: 10.1016/j.nima.2010.04.023

Abramowski, A., Aharonian, F., Ait Benkhali, F., Akhperjanian, A. G., Angüner, E. O., Backes, M., et al. (2016). Acceleration of petaelectronvolt protons in the Galactic Centre. Nature 531:476. doi: 10.1038/nature17147

Abreu, P., Aglietta, M., Ahlers, M., Ahn, E. J., Albuquerque, I. F. M., Allard, D., et al. (2011a). A search for ultra-high energy neutrinos in highly inclined events at the Pierre Auger observatory. Phys. Rev. D 84:122005. doi: 10.1103/PhysRevD.84.122005

Abreu, P., Aglietta, M., Ahlers, M., Ahn, E. J., Albuquerque, I. F. M., Allard, D., et al. (2012). Search for point-like sources of ultra-high energy neutrinos at the Pierre Auger Observatory and improved limit on the diffuse flux of tau neutrinos. Astrophys. J. 755:L4. doi: 10.1088/2041-8205/755/1/L4

Abreu, P., Aglietta, M., Ahlers, M., Ahn, E. J., Albuquerque, I. F. M., Allard, D., et al. (2013). Ultrahigh energy neutrinos at the Pierre Auger observatory. Adv. High Energy Phys. 2013:708680. doi: 10.1155/2013/708680

Abreu, P., Aglietta, M., Ahn, E. J., Albuquerque, I. F. M., Allard, D., Allekotte, I., et al. (2011b). The lateral trigger probability function for the ultra-high energy cosmic ray showers detected by the Pierre Auger Observatory. Astropart. Phys. 35, 266-276. doi: 10.1016/j.astropartphys.2011.08.001

Ackermann, M., Ajello, M., Allafort, A., Baldini, L., Ballet, J., Bastieri, D., et al. (2012). GeV observations of star-forming galaxies with Fermi LAT. Astrophys. J. 755:164. doi: 10.1088/0004-637X/755/2/164

Ackermann, M., Ajello, M., Atwood, W. B., Baldini, L., Ballet, J., Barbiellini, G., et al. (2016). 2FHL: the second catalog of hard fermi-LAT sources. Astrophys. J. Suppl. 222:5. doi: 10.3847/0067-0049/222/1/5

Adrian-Martinez, Al Samarai, I., Albert, A., Anghinolfi, M., Anton, G., Anvar, S., et al. (2013). Search for a correlation between ANTARES neutrinos and Pierre AugerObservatory UHECRs arrival directions. Astrophys. J. 774:19. doi: 10.1088/0004-637X/774/1/19

Adrian-Martinez, S., Albert, A., André, M., Anton, G., Ardid, M., Aubert, J.J., et al. (2016). High-energy neutrino follow-up search of gravitational wave event GW150914 with ANTARES and IceCube. Phys. Rev. D 93:122010. doi: 10.1103/PhysRevD.93.122010

Agostinelli, S., Allison, J., Amako, K., Apostolakies, J., Araujo, H., Arce, P., et al. (2003). GEANT4: a simulation toolkit. Nucl. Instrum. Meth. A 506, 250-303. doi: 10.1016/S0168-9002(03)01368-8

Al Samarai, I., et al. (2018). All-sky search for correlations in the arrival directions of astrophysical neutrino candidates and ultrahigh-energy cosmic rays. PoS ICRC2017:961.

Albert, A., Andre, M., Anghinolfi, M., Anton, G., Ardid, M., Aubert, J.-J., et al. (2017b). Search for high-energy neutrinos from gravitational wave event GW151226 and candidate LVT151012 with ANTARES and IceCube. Phys. Rev. D 96:022005. doi: 10.1103/PhysRevD.96.022005

Albert, A., Andre, M., Anghinolfi, M., Ardid, M., Aubert, J.-J., Aublin, J., et al. (2017a). Search for high-energy neutrinos from binary neutron star merger GW170817 with ANTARES, IceCube, and the Pierre Auger observatory. Astrophys. J. 850:L35. doi: 10.3847/2041-8213/aa9aed

Aloisio, R., Matarrese, S., and Olinto, A. V. (2015). Super heavy dark matter in light of BICEP2, planck and ultra high energy cosmic rays observations. JCAP 1508:024. doi: 10.1088/1475-7516/2015/08/024

Alvarez Muñiz, J. (2018). Multi-messenger astrophysics at ultra-high energy with the Pierre Auger observatory. Eur. Phys. J. Web of Conf.

Alves Batista, R., Dundovic, A., Erdmann, M., Kampert, K.-H., Kuempel, D., Müller, G., et al. (2016). CRPropa 3 - a public astrophysical simulation framework for propagating extraterrestrial ultra-high energy particles. JCAP 1605:038.

Aramo, C., Insolia, A., Leonardi, A., Miele, G., Perrone, L., Pisanti, O., et al. (2005). Earth-skimming UHE Tau neutrinos at the fluorescence detector of Pierre Auger Observatory. Astropart. Phys. 23, 65-77. doi: 10.1016/j.astropartphys.2004.11.008

Argiro, S., Barroso, S. L. C., Gonzalez, J., Nellen, L., Paul, T. C., Porter, T. A., et al. (2007). The offline software framework of the Pierre Auger observatory. Nucl. Instrum. Meth. A 580, 1485-1496. doi: 10.1016/j.nima.2007.07.010

Athar, H., Jezabek, M., and Yasuda, O. (2000). Effects of neutrino mixing on high-energy cosmic neutrino flux. Phys. Rev. D 62:103007. doi: 10.1103/PhysRevD.62.103007

Aublin, J., Capone, A., and Di Palma, I. (2018). "Search for a correlation between ANTARES high-energy neutrinos and ultra high-energy cosmic rays detected by the Pierre Auger Observatory and the Telescope Array" in PoS ICRC2017 (Busan). doi: 10.22323/1.301.0990

Ave, M., Vazquez, R. A., and Zas, E. (2000). Modelling horizontal air showers induced by cosmic rays. Astropart. Phys. 14:91. doi: 10.1016/S0927-6505(00)00113-4

Barwick, S. W. (2000). High-energy cosmic neutrinos. Phys. Scripta T 85, 106-116. doi: 10.1238/Physica.Topical.085a00106

Bellido, J. (2018). "Depth of maximum of air-shower profiles at the Pierre Auger Observatory: measurements above $10^{17.2} \mathrm{eV}$ and Composition Implications" in PoS ICRC2017 (Busan). doi: 10.22323/1.301.0506

Berezinsky, V. S., and Smirnov, A. Yu. (1975). Cosmic neutrinos of ultrahigh energies and detection possibility. Astrophys. Space Sci. 32, 461-482. doi: 10.1007/BF00643157

Bhattacharjee, P., and Sigl, G. (2000). Origin and propagation of extremely high-energy cosmic rays. Phys. Rept. 327, 109-247. doi: 10.1016/S0370-1573(99)00101-5

Bionta, R. M., Blewitt, G., Bratton, C. B., Casper, D., Ciocio, A., Claus, R., et al. (1987). Observation of a neutrino burst in coincidence with supernova SN 1987A in the large magellanic cloud. Phys. Rev. Lett. 58:1494. doi: 10.1103/PhysRevLett.58.1494

Bleve, C. (2016). Updates on the neutrino and photon limits from the Pierre Auger Observatory. PoS ICRC2015:1103. doi: 10.22323/1.236.1103

Bray, J. D., and Scaife, A. M. M. (2018). An upper limit on the strength of the extragalactic magnetic field from ultra-high-energy cosmic-ray anisotropy. Astrophys. J. 861:3. doi: 10.3847/1538-4357/aac777

Capelle, K. S., Cronin, J. W., Parente, G., and Zas, E. (1998). On the detection of ultrahigh-energy neutrinos with the Auger Observatory. Astropart. Phys. 8, 321-328. doi: 10.1016/S0927-6505(97)00059-5

Coulter, D. A., Foley, R. J., Kilpatrick, C. D., Drout, M. R., Piro, A. L., Shappee, B. J., et al. (2017). Swope supernova survey 2017a (SSS17a), the optical counterpart to a gravitational wave source. Science. $358,1556-1558$. doi: $10.1126 /$ science. aap9811

Crocker, R. M., Fatuzzo, M., Jokipii, R., Melia, F., and Volkas, R. R. (2005). The AGASA/SUGAR anisotropies and $\mathrm{TeV}$ gamma rays from the Galactic Center: a possible signature of extremely high-energy neutrons. Astrophys. J. 622, 892-909. doi: 10.1086/427972

De Angelis, A., Galanti, G., and Roncadelli, M. (2013). Transparency of the universe to gamma rays. Mon. Not. Roy. Astron. Soc. 432, 3245-3249. doi: $10.1093 / \mathrm{mnras} / \mathrm{stt} 684$

Dermer, C. D., and Razzaque, S. (2010). Acceleration of ultra-high energy cosmic rays in the colliding shells of blazars and GRBs: constraints from the Fermi Gamma ray space telescope. Astrophys. J. 724, 1366-1372. doi: 10.1088/0004-637X/724/2/1366

Engel, R. (2016). Upgrade of the Pierre Auger observatory (AugerPrime). PoS ICRC2015:686.

Erber, T. (1966). High-energy electromagnetic conversion processes in intense magnetic fields. Rev. Mod. Phys. 38, 626-659. doi: 10.1103/RevModPhys.38.626

Fang, K., and Metzger, B. D. (2017). High-energy neutrinos from millisecond magnetars formed from the merger of binary neutron stars. Astrophys. J. 849:153. doi: 10.3847/1538-4357/aa8b6a

Fargion, D. (2002). Discovering ultra high energy neutrinos by horizontal and upward tau air-showers: evidences in terrestrial gamma flashes? Astrophys. J. 570, 909-925. doi: 10.1086/339772

Feldman, G. J., and Cousins, R. D. (1998). A Unified approach to the classical statistical analysis of small signals. Phys. Rev. D 57, 3873-3889. doi: 10.1103/PhysRevD.57.3873

Fisher, R. A. (1936). The use of multiple measurements in taxonomic problems. Annals Eugen. 7, 179-188. doi: 10.1111/j.1469-1809.1936.tb02137.x 
Gaisser, T. K., Halzen, F., and Stanev, T. (1995). Particle astrophysics with high-energy neutrinos. Phys. Rept. 258, 173-236. doi: 10.1016/0370-1573(95)00003-Y

Gao, H., Zhang, B., Wu, X.-F., and Dai, Z.-G. (2013). Possible high-energy neutrino and photon signals from gravitational wave bursts due to double neutron star mergers. Phys. Rev. D 88:043010. doi: 10.1103/PhysRevD.88.043010

Goldstein, A., Veres, P., Burns, E., Briggs, M. S., Hamburg, R., Kocevski, D., et al. (2017). An ordinary short gamma-ray burst with extraordinary implications: Fermi-GBM detection of GRB 170817A. Astrophys. J. 848:L14. doi: $10.3847 / 2041-8213 /$ aa $8 f 41$

Halzen, F. (2003). "Multi-messenger astronomy: cosmic rays, gamma-rays, and neutrinos," in Proceedings, 21st Texas Symposium on Relativistic Astrophysics (Texas in Tuscany): December 9-13, 2002 (Florence), 117-131.

Heck, D., Knapp, J., Capdevielle, J. N., Schatz, G., and Thouw, T. (2000). CORSIKA: A Monte Carlo Code to Simulate Extensive Air Showers, Forschungszentrum Karlsruhe-Wissenschaftliche Berichte. Available online at: https://web.ikp.kit.edu/corsika/

Hillas, A. M. (1984). The origin of ultrahigh-energy cosmic rays. Ann. Rev. Astron. Astrophys. 22, 425-444. doi: 10.1146/annurev.aa.22.090184.002233

Hillas, A. M., Hollows, J. D., Hunter, H. W., and Marsden, D. J. (1969). "Calculations on the particle and energy-loss densities in extensive air showers at large axial distances," in Proceedings, 11th International Cosmic Ray Conference: Hungary, August 25-September 4, 1969, Vol. 3, 533-538.

Hinton, J. A., and Hofmann, W. (2009). Teraelectronvolt astronomy. Ann. Rev. Astron. Astrophys. 47, 523-565. doi: 10.1146/annurev-astro-082708-101816

Hirata, K., Kajita, T., Koshiba, M., Nakahata, M., Oyama, Y., Sato, N., et al. (1987). Observation of a neutrino burst from the Supernova SN 1987a. Phys. Rev. Lett. 58, 1490-1493. doi: 10.1103/PhysRevLett.58.1490

Homola, P., Risse, M., Engel, R., Gora, D., Pekala, J., Wilczynska, B., et al. (2007). Characteristics of geomagnetic cascading of ultrahigh energy photons at the southern and northern sites of the Pierre Auger Observatory. Astropart. Phys. 27, 174-184. doi: 10.1016/j.astropartphys.2006.10.005

Hörandel, J. (2018). Precision measurements of cosmic rays up to the highest energies with a large radio array at the Pierre Auger Observatory. Eur. Phys. J. Web of Conf.

Kalashev, O. K., and Kuznetsov, M. Yu. (2016). Constraining heavy decaying dark matter with the high energy gamma-ray limits. Phys. Rev. D 94:063535. doi: 10.1103/PhysRevD.94.063535

Kimura, S. S., Murase, K., Mészàros, P., and Kiuchi, K. (2017). Highenergy neutrino emission from short gamma-ray bursts: prospects for coincident detection with gravitational waves. Astrophys. J. 848:L4. doi: 10.3847/2041-8213/aa8d14

Kotera, K., and Silk, J. (2016). Ultrahigh energy cosmic rays and black hole mergers. Astrophys. J. 823:L29. doi: 10.3847/2041-8205/823/2/L29

Landau, L. D., and Pomeranchuk, I. (1953). Limits of applicability of the theory of bremsstrahlung electrons and pair production at high-energies. Dokl. Akad. Nauk Ser. Fiz. 92, 535-536.

Learned, J. G., and Pakvasa, S. (1995). Detecting tau-neutrino oscillations at PeV energies. Astropart. Phys. 3, 267-274. doi: 10.1016/0927-6505(94)00043-3

Lemoine, M., Sigl, G., Olinto, A. V., and Schramm, D. N. (1997). Ultrahigh-energy cosmic ray sources and large scale magnetic fields. Astrophys. J. 486, L115-L118. doi: $10.1086 / 310847$

Letessier-Selvon, A. (2001). Establishing the GZK cutoff with ultrahigh-energy tau neutrinos. AIP Conf. Proc. 566, 157-171. doi: 10.1063/1.1378629

LIGO-Collaboration (2018). LIGO Document P1800381-v2. Available online at: https://dcc.ligo.org/LIGO-P1800381/public

Martello, D. (2017). The Pierre Auger Observatory status and the AugerPrime upgrade program. EPJ Web Conf. 145:05001. doi: 10.1051/epjconf/201714505001
Matthews, J. H., Bell, A. R., Blundell, K. M., and Araudo, A. T. (2019). Ultra-high energy cosmic rays from shocks in the lobes of powerful radio galaxies. Mon. Not. Roy. Astron. Soc. 482:4303. doi: 10.1093/mnras/sty2936

McBreen, B., and Lambert, C. J. (1981). Interactions of High-energy ( $E>5 \times$ $10^{19} \mathrm{eV}$ ) Photons in the Earth's Magnetic Field. Phys. Rev. D 24, 2536-2538. doi: 10.1103/PhysRevD.24.2536

Mészáros, P. (2006). Gamma-ray bursts. Rept. Prog. Phys. 69, 2259-2322. doi: 10.1088/0034-4885/69/8/R01

Metzger, B. D. (2017). Kilonovae. Living Rev. Rel. 20:3. doi: 10.1007/s41114-017-0006-z

Migdal, A. B. (1956). Bremsstrahlung and pair production in condensed media at high-energies. Phys. Rev. 103, 1811-1820. doi: 10.1103/PhysRev.103. 1811

Moharana, R., Razzaque, S., Gupta, N., and Mészáros, P. (2016). High energy neutrinos from the gravitational wave event GW150914 possibly associated with a short Gamma-Ray Burst. Phys. Rev. D 93:123011. doi: 10.1103/PhysRevD.93.123011

Mollerach, S., and Roulet, E. (2018). Progress in high-energy cosmic ray physics. Prog. Part. Nucl. Phys. 98, 85-118. doi: 10.1016/j.ppnp.2017. 10.002

Murase, K., Kashiyama, K., Mászáros, P., Shoemaker, I., and Senno, N. (2016). Ultrafast outflows from black hole mergers with a minidisk. Astrophys. J. 822:L9. doi: 10.3847/2041-8205/822/1/L9

Perna, R., Lazzati, D., and Giacomazzo, B. (2016). Short gamma-ray bursts from the merger of two black holes. Astrophys. J. 821:L18. doi: 10.3847/2041-8205/821/1/L18

Risse, M., and Homola, P. (2007). Search for ultrahigh energy photons using air showers. Mod. Phys. Lett. A 22, 749-766. doi: 10.1142/S02177323070 22864

Romero, G. E., Müller, A. L., and Roth, M. (2018). Particle acceleration in the superwinds of starburst galaxies. Astron. Astrophys. 616:A57. doi: 10.1051/0004-6361/201832666

Savchenko, V., Ferrigno, C., Kuulkers, E., Bazzano, A., Bozzo, E., Brandt, S., et al. (2017). INTEGRAL detection of the first prompt gamma-ray signal coincident with the gravitational-wave event GW170817. Astrophys. J. 848:L15. doi: 10.3847/2041-8213/aa8f94

Sciutto, S. (1999). AIRES: A System for Air Shower Simulations. User's Guide and Reference Manual, Version 2.2.0. Available online at: http://www2.fisica.unlp. edu.ar/auger/aires/eg_Aires.html

Smith, M. W. E., Fox, D. B., Cowen, D. F., Mészáros, P., Tešic, G., Fixelle, J., et al. (2013). The astrophysical multimessenger observatory network (AMON) Astropart. Phys. 45, 56-70. doi: 10.1016/j.astropartphys.2013.03.003

Zas, E. (2005). Neutrino detection with inclined air showers. New J. Phys. 7:130. doi: $10.1088 / 1367-2630 / 7 / 1 / 130$

Zas, E. (2018). "Searches for neutrino fluxes in the $\mathrm{EeV}$ regime with the Pierre Auger Observatory" in PoS ICRC2017 (Busan). doi: 10.22323/1. 301.0972

Conflict of Interest Statement: The authors declare that the research was conducted in the absence of any commercial or financial relationships that could be construed as a potential conflict of interest.

Copyright (c) 2019 Kampert, Alejandro Mostafa, Zas and The Pierre Auger Collaboration. This is an open-access article distributed under the terms of the Creative Commons Attribution License (CC BY). The use, distribution or reproduction in other forums is permitted, provided the original author(s) and the copyright owner(s) are credited and that the original publication in this journal is cited, in accordance with accepted academic practice. No use, distribution or reproduction is permitted which does not comply with these terms. 


\section{A. APPENDIX}

\section{A.1 Full Author List and Affiliations}

A. $\mathrm{Aab}^{75}, \mathrm{P} . \mathrm{Abreu}^{67}$, M. Aglietta ${ }^{50,49}$, I.F.M. Albuquerque ${ }^{19}$, J.M. Albury ${ }^{12}$, I. Allekotte ${ }^{1}$, A. Almela ${ }^{8,11}$, J. Alvarez Castillo ${ }^{63}$, J. Alvarez-Muñiz ${ }^{74}$, G.A. Anastasi ${ }^{42,43}$, L. Anchordoqui ${ }^{82}$, B. Andrada $^{8}$, S. Andringa ${ }^{67}$, C. Aramo ${ }^{47}$, H. Asorey ${ }^{1,28}$, P. Assis ${ }^{67}$, G. Avila ${ }^{9,10}$, A.M. Badescu ${ }^{70}$, A. Bakalova ${ }^{30}$, A. Balaceanu ${ }^{68}$, F. Barbato ${ }^{56,47}$, R.J. Barreira Luz ${ }^{67}$, S. Baur ${ }^{37}$, K.H. Becker ${ }^{35}$, J.A. Bellido ${ }^{12}$, C. Berat ${ }^{34}$, M.E. Bertaina ${ }^{58,49}$, X. Bertou ${ }^{1}$, P.L. Biermann ${ }^{b}$, J. Biteau ${ }^{32}$, S.G. Blaess ${ }^{12}$, A. Blanco ${ }^{67}$, J. Blazek ${ }^{30}$, C. Bleve $e^{52,45}$, M. Boháčová ${ }^{30}$, D. Boncioli ${ }^{42,43}$, C. Bonifazi ${ }^{24}$, N. Borodai ${ }^{64}$, A.M. Botti ${ }^{8,37}$, J. Brack ${ }^{e}$, T. Bretz ${ }^{39}$, A. Bridgeman ${ }^{36}$, F.L. Briechle ${ }^{39}$, P. Buchholz ${ }^{41}$, A. Bueno ${ }^{73}$, S. Buitink ${ }^{14}$, M. Buscemi ${ }^{54,44}$, K.S. Caballero-Mora ${ }^{62}$, L. Caccianiga ${ }^{55}$, L. Calcagni ${ }^{4}$, A. Cancio ${ }^{11,8}$, F. Canfora ${ }^{75,77}$, J.M. Carceller ${ }^{73}$, R. Caruso ${ }^{54,44}$, A. Castellina ${ }^{50,49}$, F. Catalani ${ }^{17}$, G. Cataldi ${ }^{45}$, L. Cazon ${ }^{67}$, M. Cerda ${ }^{9}$, J.A. Chinellato ${ }^{20}$, J. Chudoba ${ }^{30}$, L. Chytka ${ }^{31}$, R.W. Clay $^{12}$, A.C. Cobos Cerutti ${ }^{7}$, R. Colalillo ${ }^{56,47}$, A. Coleman ${ }^{86}$, M.R. Coluccia ${ }^{52,45}$, R. Conceição ${ }^{67}$, A. Condorellii' ${ }^{42,43}$, G. Consolati ${ }^{46,51}$, F. Contreras ${ }^{9,10}$, F. Convenga ${ }^{52,45}$, M.J. Cooper ${ }^{12}$, S. Coutu ${ }^{86}$, C.E. Covault ${ }^{80}$, B. Daniel ${ }^{20}$, S. Dasso ${ }^{5,3}$, K. Daumiller ${ }^{37}$, B.R. Dawson ${ }^{12}$, J.A. Day ${ }^{12}$, R.M. de Almeida ${ }^{26}$, S.J. de Jong ${ }^{75,77}$, G. De Mauro ${ }^{75,77}$, J.R.T. de Mello Neto ${ }^{24,25}$, I. De Mitri ${ }^{42,43}$, J. de Oliveira ${ }^{26}$, F.O. de Oliveira Salles ${ }^{15}$, V. de Souza ${ }^{18}$, J. Debatin ${ }^{36}$, M. del Río ${ }^{10}$, O. Deligny ${ }^{32}$, N. Dhital ${ }^{64}$, M.L. Díaz Castro ${ }^{20}$, F. Diogo ${ }^{67}$, C. Dobrigkeit ${ }^{20}$, J.C. D’Olivo ${ }^{63}$, Q. Dorosti ${ }^{41}$, R.C. dos Anjos ${ }^{23}$, M.T. Dova ${ }^{4}$, A. Dundovic ${ }^{40}$, J. Ebr ${ }^{30}$, R. Engel ${ }^{36,37}$, M. Erdmann ${ }^{39}$, C.O. Escobar ${ }^{c}$, A. Etchegoyen ${ }^{8,11}$, H. Falcke ${ }^{75,78,77}$, J. Farmer ${ }^{87}$, G. Farrar ${ }^{85}$, A.C. Fauth ${ }^{20}$, N. Fazzini ${ }^{c}$, F. Feldbusch ${ }^{38}$, F. Fenu ${ }^{58,49}$, L.P. Ferreyro ${ }^{8}$, J.M. Figueira ${ }^{8}$, A. Filipčič ${ }^{72,71}$, M.M. Freire ${ }^{6}$, T. Fujii ${ }^{87, f}$, A. Fuster ${ }^{8,11}$, B. García ${ }^{7}$, H. Gemmeke ${ }^{38}$, A. Gherghel-Lascu ${ }^{68}$, P.L. Ghia ${ }^{32}$, U. Giaccari ${ }^{15}$, M. Giammarchi ${ }^{46}$, M. Giller ${ }^{65}$, D. Głas ${ }^{66}$, J. Glombitza ${ }^{39}$, F. Gobbi ${ }^{9}$, G. Golup ${ }^{1}$, M. Gómez Berisso $^{1}$, P.F. Gómez Vitale ${ }^{9,10}$, J.P. Gongora ${ }^{9}$, N. González ${ }^{8}$, I. Goos ${ }^{1,37}$, D. Góra ${ }^{64}$, A. Gorgi ${ }^{50,49}$, M. Gottowik ${ }^{35}$, T.D. Grubb ${ }^{12}$, F. Guarino ${ }^{56,47}$, G.P. Guedes ${ }^{21}$, E. Guido ${ }^{49,58}$, R. Halliday ${ }^{80}$, M.R. Hampel ${ }^{8}$, P. Hansen ${ }^{4}$, D. Harari ${ }^{1}$, T.A. Harrison ${ }^{12}$, V.M. Harvey ${ }^{12}$, A. Haungs ${ }^{37}$, T. Hebbeker ${ }^{39}$, D. Heck ${ }^{37}$, P. Heimann ${ }^{41}$, G.C. Hill ${ }^{12}$, C. Hojvat ${ }^{c}$, E.M. Holt ${ }^{36,8}$, P. Homola ${ }^{64}$, J.R. Hörandel ${ }^{75,77}$, P. Horvath ${ }^{31}$, M. Hrabovský ${ }^{31}$,

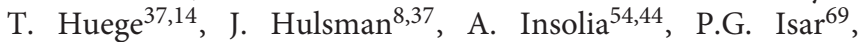
I. Jandt ${ }^{35}$, J.A. Johnsen ${ }^{81}$, M. Josebachuili ${ }^{8}$, J. Jurysek ${ }^{30}$, A. Kääpä ${ }^{35}$, K.H. Kampert ${ }^{35}$, B. Keilhauer ${ }^{37}$, N. Kemmerich ${ }^{19}$, J. Kemp ${ }^{39}$, H.O. Klages ${ }^{37}$, M. Kleifges ${ }^{38}$, J. Kleinfeller ${ }^{9}$, R. Krause ${ }^{39}$, D. Kuempel ${ }^{35}$, G. Kukec Mezek ${ }^{71}$, A. Kuotb Awad $^{36}$, B.L. Lago ${ }^{16}$, D. LaHurd ${ }^{80}$, R.G. Lang ${ }^{18}$, R. Legumina ${ }^{65}$, M.A. Leigui de Oliveira ${ }^{22}$, V. Lenok ${ }^{37}$, A. Letessier-Selvon ${ }^{33}$, I. Lhenry-Yvon ${ }^{32}$, O.C. Lippmann ${ }^{15}$, D. Lo Presti ${ }^{54,44}$, L. Lopes ${ }^{67}$, R. López ${ }^{59}$, A. López Casado ${ }^{74}$, R. Lorek $^{80}$, Q. Luce ${ }^{32}$, A. Lucero ${ }^{8}$, M. Malacari ${ }^{87}$, G. Mancarella ${ }^{52,45}$, D. Mandat ${ }^{30}$, B.C. Manning ${ }^{12}$, P. Mantsch ${ }^{c}$, A.G. Mariazzi ${ }^{4}$, I.C. Mariş ${ }^{13}$, G. Marsella ${ }^{52,45}$, D. Martello ${ }^{52,45}$, H. Martinez ${ }^{60}$, O. Martínez Bravo ${ }^{59}$, M. Mastrodicasa ${ }^{53,43}$, H.J. Mathes ${ }^{37}$, S. Mathys ${ }^{35}$, J. Matthews ${ }^{83}$,
G. Matthiae ${ }^{57,48}$, E. Mayotte ${ }^{35}$, P.O. Mazurc ${ }^{c}$, G. Medina-Tanco ${ }^{63}$, D. $M_{\text {elo }}^{8}$, A. Menshikov ${ }^{38}$, K.-D. Merenda ${ }^{81}$, S. Michal ${ }^{31}$, M.I. Micheletti ${ }^{6}$, L. Middendorf ${ }^{39}$, L. Miramonti ${ }^{55,46}$, B. Mitrica ${ }^{68}$, D. Mockler ${ }^{36}$, S. Mollerach ${ }^{1}$, F. Montanet ${ }^{34}$ C. Morello ${ }^{50,49}$, G. Morlino ${ }^{42,43}$, M. Mostafá ${ }^{86}$, A.L. Müller ${ }^{8,37}$ M.A. Muller ${ }^{20, d}$, S. Müller ${ }^{36,8}$, R. Mussa ${ }^{49}$, L. Nellen ${ }^{63}$, P.H. Nguyen ${ }^{12}$, M. Niculescu-Oglinzanu ${ }^{68}$, M. Niechciol ${ }^{41}$, D. Nitz ${ }^{84, g}$, D. Nosek ${ }^{29}$, V. Novotny ${ }^{29}$, L. Nožka ${ }^{31}$, A Nucita $^{52,45}$, L.A. Núñez ${ }^{28}$, F. Oikonomou ${ }^{86, i}$, A. Olinto ${ }^{87}$, M. Palatka ${ }^{30}$ J. Pallotta ${ }^{2}$, M.P. Panetta ${ }^{52,45}$, P. Papenbreer ${ }^{35}$, G. Parente ${ }^{74}$, A. Parra ${ }^{59}$, M. Pech ${ }^{30}$, F. Pedreira ${ }^{74}$, J. Pȩkala ${ }^{64}$, R. Pelayo ${ }^{61}$, J. Peña-Rodriguez ${ }^{28}$, L.A.S. Pereira ${ }^{20}$, M. Perlin ${ }^{8}$, L. Perrone ${ }^{52,45}$, C. Peters $^{39}$, S. Petrera ${ }^{42,43}$, J. Phuntsok ${ }^{86}$, T. Pierog ${ }^{37}$,

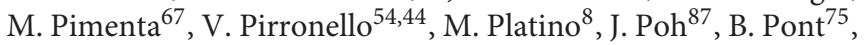
C. Porowski ${ }^{64}$, R.R. Prado ${ }^{18}$, P. Privitera ${ }^{87}$, M. Prouza ${ }^{30}$, A. Puyleart ${ }^{84}$, S. Querchfeld ${ }^{35}$, S. Quinn ${ }^{80}$, R. Ramos-Pollan ${ }^{28}$, J. Rautenberg ${ }^{35}$, D. Ravignani ${ }^{8}$, M. Reininghaus ${ }^{37}$, J. Ridky ${ }^{30}$, F. Riehn ${ }^{67}$, M. Risse ${ }^{41}$, P. Ristori ${ }^{2}$, V. Rizi ${ }^{53,43}$, W. Rodrigues de Carvalho $^{19}$, J. Rodriguez Rojo ${ }^{9}$, M.J. Roncoroni ${ }^{8}$, M. Roth $^{37}$, E. Roulet ${ }^{1}$, A.C. Rovero ${ }^{5}$, P. Ruehl ${ }^{41}$, S.J. Saffi ${ }^{12}$, A. Saftoiu ${ }^{68}$, F. Salamida ${ }^{53,43}$, H. Salazar ${ }^{59}$, G. Salina ${ }^{48}$, J.D. Sanabria Gomez ${ }^{28}$, F. Sánchez ${ }^{8}$, E.M. Santos ${ }^{19}$, E. Santos ${ }^{30}$, F. Sarazin ${ }^{81}$, R. Sarmento ${ }^{67}$, C. Sarmiento-Cano ${ }^{8}$, R. Sato ${ }^{9}$, P. Savina ${ }^{52,45}$, M. Schauer ${ }^{35}$, V. Scherini ${ }^{45}$, H. Schieler ${ }^{37}$, M. Schimassek ${ }^{36}$, M. Schimp ${ }^{35}$, F. Schlüter ${ }^{37}$, D. Schmidt ${ }^{36}$, O. Scholten ${ }^{76,14}$, P. Schovánek ${ }^{30}$, F.G. Schröder ${ }^{88,37}$, S. Schröder ${ }^{35}$, J. Schumacher ${ }^{39}$, S.J. Sciutto ${ }^{4}$, M. Scornavacche ${ }^{8}$, R.C. Shellard ${ }^{15}$, G. Sigl ${ }^{40}$, G. Silli ${ }^{8,37}$, O. Sima ${ }^{68, h}$, R. Šmída ${ }^{87}$, G.R. Snow ${ }^{89}$, P. Sommers ${ }^{86}$, J.F. Soriano ${ }^{82}$, J. Souchard ${ }^{34}$, R. Squartini ${ }^{9}$, D. Stanca ${ }^{68}$, S. Stanic ${ }^{71}$, J. Stasielak ${ }^{64}$, P. Stassi ${ }^{34}$, M. Stolpovskiy ${ }^{34}$, A. Streich ${ }^{36}$, F. Suarez ${ }^{8,11}$, M. Suárez-Durán ${ }^{28}$, T. Sudholz ${ }^{12}$, T. Suomijärvi ${ }^{32}$, A.D. Supanitsky ${ }^{8}$, J. Šupík ${ }^{31}$, Z. Szadkowski ${ }^{66}$, A. Taboada ${ }^{37}$, O.A. Taborda ${ }^{1}$, A. Tapia ${ }^{27}$, C. Timmermans ${ }^{77,75}$, C.J. Todero Peixoto ${ }^{17}$, B. Tomé ${ }^{67}$, G. Torralba Elipe ${ }^{74}$, A. Travaini ${ }^{9}$, P. Travnicek ${ }^{30}$, M. Trini ${ }^{71}$, M. Tueros ${ }^{4}$, R. Ulrich ${ }^{37}$, M. Unger ${ }^{37}$, M. Urban ${ }^{39}$, J.F. Valdés Galicia $^{63}$, I. Valiño ${ }^{42,43}$, L. Valore ${ }^{56,47}$, P. van Bodegom ${ }^{12}$, A.M. van den Berg $^{76}$, A. van Vliet ${ }^{75}$, E. Varela ${ }^{59}$, B. Vargas Cárdenas $^{63}$, D. Veberič ${ }^{37}$, C. Ventura ${ }^{25}$, I.D. Vergara Quispe ${ }^{4}$, V. Verzi ${ }^{48}$, J. Vicha ${ }^{30}$, L. Villaseñor ${ }^{59}$, J. Vink ${ }^{79}$, S. Vorobiov ${ }^{71}$, H. Wahlberg ${ }^{4}$, A.A. Watson ${ }^{a}$, M. Weber ${ }^{38}$, A. Weindl ${ }^{37}$, M. Wiedeński ${ }^{66}$, L. Wiencke ${ }^{81}, \mathrm{H}$. Wilczyński ${ }^{64}$, T. Winchen ${ }^{14}$, M. Wirtz ${ }^{39}$, D. Wittkowski ${ }^{35}$, B. Wundheiler ${ }^{8}$, L. Yang ${ }^{71}$, A. Yushkov ${ }^{30}$, E. Zas ${ }^{74}$, D. Zavrtanik ${ }^{71,72}$, M. Zavrtanik ${ }^{72,71}$, L. Zehrer ${ }^{71}$, A. Zepeda ${ }^{60}$, B. Zimmermann ${ }^{37}$, M. Ziolkowski ${ }^{41}$, Z. Zong ${ }^{32}$, F. Zuccarello ${ }^{54,44}$

1 Centro Atómico Bariloche and Instituto Balseiro (CNEAUNCuyo-CONICET), San Carlos de Bariloche, Argentina

2 Centro de Investigaciones en Láseres y Aplicaciones, CITEDEF and CONICET, Villa Martelli, Argentina

${ }^{3}$ Departamento de Física and Departamento de Ciencias de la Atmósfera y los Océanos, FCEyN, Universidad de Buenos Aires and CONICET, Buenos Aires, Argentina

${ }^{4}$ IFLP, Universidad Nacional de La Plata and CONICET, La Plata, Argentina

${ }^{5}$ Instituto de Astronomía y Física del Espacio (IAFE, CONICETUBA), Buenos Aires, Argentina 
6 Instituto de Física de Rosario (IFIR) - CONICET/U.N.R. and Facultad de Ciencias Bioquímicas y Farmacéuticas U.N.R., Rosario, Argentina

7 Instituto de Tecnologías en Detección y Astropartículas (CNEA, CONICET, UNSAM), and Universidad Tecnológica Nacional - Facultad Regional Mendoza (CONICET/CNEA), Mendoza, Argentina

8 Instituto de Tecnologías en Detección y Astropartículas (CNEA, CONICET, UNSAM), Buenos Aires, Argentina

${ }^{9}$ Observatorio Pierre Auger, Malargüe, Argentina

${ }^{10}$ Observatorio Pierre Auger and Comisión Nacional de Energía Atómica, Malargüe, Argentina

11 Universidad Tecnológica Nacional - Facultad Regional Buenos Aires, Buenos Aires, Argentina

12 University of Adelaide, Adelaide, S.A., Australia

13 Université Libre de Bruxelles (ULB), Brussels, Belgium

${ }^{14}$ Vrije Universiteit Brussels, Brussels, Belgium

15 Centro Brasileiro de Pesquisas Fisicas, Rio de Janeiro, RJ, Brazil

16 Centro Federal de Educação Tecnológica Celso Suckow da Fonseca, Nova Friburgo, Brazil

17 Universidade de São Paulo, Escola de Engenharia de Lorena, Lorena, SP, Brazil

18 Universidade de São Paulo, Instituto de Física de São Carlos, São Carlos, SP, Brazil

19 Universidade de São Paulo, Instituto de Física, São Paulo, SP, Brazil

${ }^{20}$ Universidade Estadual de Campinas, IFGW, Campinas, SP, Brazil

${ }^{21}$ Universidade Estadual de Feira de Santana, Feira de Santana, Brazil

22 Universidade Federal do ABC, Santo André, SP, Brazil

23 Universidade Federal do Paraná, Setor Palotina, Palotina, Brazil

24 Universidade Federal do Rio de Janeiro, Instituto de Física, Rio de Janeiro, RJ, Brazil

${ }^{25}$ Universidade Federal do Rio de Janeiro (UFRJ), Observatório do Valongo, Rio de Janeiro, RJ, Brazil

${ }^{26}$ Universidade Federal Fluminense, EEIMVR, Volta Redonda, RJ, Brazil

27 Universidad de Medellín, Medellín, Colombia

${ }^{28}$ Universidad Industrial de Santander, Bucaramanga, Colombia

29 Charles University, Faculty of Mathematics and Physics, Institute of Particle and Nuclear Physics, Prague, Czech Republic

${ }^{30}$ Institute of Physics of the Czech Academy of Sciences, Prague, Czech Republic

${ }^{31}$ Palacky University, RCPTM, Olomouc, Czech Republic

32 Institut de Physique Nucléaire d'Orsay (IPNO), Université Paris-Sud, Univ. Paris/Saclay, CNRS-IN2P3, Orsay, France

33 Laboratoire de Physique Nucléaire et de Hautes Energies (LPNHE), Universités Paris 6 et Paris 7, CNRS-IN2P3, Paris, France

34 Univ. Grenoble Alpes, CNRS, Grenoble Institute of Engineering Univ. Grenoble Alpes, LPSC-IN2P3, 38000 Grenoble, France, France

35 Bergische Universität Wuppertal, Department of Physics, Wuppertal, Germany
${ }^{36}$ Karlsruhe Institute of Technology, Institute for Experimental Particle Physics (ETP), Karlsruhe, Germany

37 Karlsruhe Institute of Technology, Institut für Kernphysik, Karlsruhe, Germany

38 Karlsruhe Institute of Technology, Institut für Prozessdatenverarbeitung und Elektronik, Karlsruhe, Germany

39 RWTH Aachen University, III. Physikalisches Institut A, Aachen, Germany

40 Universität Hamburg, II. Institut für Theoretische Physik, Hamburg, Germany

41 Universität Siegen, Fachbereich 7 Physik - Experimentelle Teilchenphysik, Siegen, Germany

${ }^{4}$ Gran Sasso Science Institute, L’Aquila, Italy

43 INFN Laboratori Nazionali del Gran Sasso, Assergi (L'Aquila), Italy

44 INFN, Sezione di Catania, Catania, Italy

45 INFN, Sezione di Lecce, Lecce, Italy

${ }^{46}$ INFN, Sezione di Milano, Milano, Italy

47 INFN, Sezione di Napoli, Napoli, Italy

48 INFN, Sezione di Roma “Tor Vergata”, Roma, Italy

49 INFN, Sezione di Torino, Torino, Italy

${ }^{50}$ Osservatorio Astrofisico di Torino (INAF), Torino, Italy

51 Politecnico di Milano, Dipartimento di Scienze e Tecnologie

Aerospaziali, Milano, Italy

52 Università del Salento, Dipartimento di Matematica e Fisica "E. De Giorgi", Lecce, Italy

53 Università dell'Aquila, Dipartimento di Scienze Fisiche e Chimiche, L'Aquila, Italy

54 Università di Catania, Dipartimento di Fisica e Astronomia, Catania, Italy

55 Università di Milano, Dipartimento di Fisica, Milano, Italy

56 Università di Napoli "Federico II", Dipartimento di Fisica "Ettore Pancini", Napoli, Italy

57 Università di Roma "Tor Vergata”, Dipartimento di Fisica, Roma, Italy

58 Università Torino, Dipartimento di Fisica, Torino, Italy

${ }^{59}$ Benemérita Universidad Autónoma de Puebla, Puebla, México

60 Centro de Investigación y de Estudios Avanzados del IPN (CINVESTAV), México, D.F., México

61 Unidad Profesional Interdisciplinaria en Ingeniería y Tecnologías Avanzadas del Instituto

Politécnico Nacional (UPIITA-IPN), México, D.F., México

${ }^{62}$ Universidad Autónoma de Chiapas, Tuxtla Gutiérrez, Chiapas, México

63 Universidad Nacional Autónoma de México, México, D.F., México

${ }^{64}$ Institute of Nuclear Physics PAN, Krakow, Poland

65 University of Łódź, Faculty of Astrophysics, Łódź, Poland

${ }^{66}$ University of Łódź, Faculty of High-Energy Astrophysics, Łódź, Poland

67 Laboratório de Instrumentação e Física Experimental de Partículas - LIP and Instituto Superior Técnico - IST, Universidade de Lisboa - UL, Lisboa, Portugal

68 "Horia Hulubei" National Institute for Physics and Nuclear Engineering, Bucharest-Magurele, Romania

${ }^{69}$ Institute of Space Science, Bucharest-Magurele, Romania

${ }^{70}$ University Politehnica of Bucharest, Bucharest, Romania 
71 Center for Astrophysics and Cosmology (CAC), University of Nova Gorica, Nova Gorica, Slovenia

${ }^{72}$ Experimental Particle Physics Department, J. Stefan Institute, Ljubljana, Slovenia

${ }^{73}$ Universidad de Granada and C.A.F.P.E., Granada, Spain

74 Instituto Galego de Física de Altas Enerxías (I.G.F.A.E.), Universidad de Santiago de Compostela, Santiago de Compostela, Spain

75 IMAPP, Radboud University Nijmegen, Nijmegen, The Netherlands

${ }^{76}$ KVI - Center for Advanced Radiation Technology, University of Groningen, Groningen, The Netherlands

77 Nationaal Instituut voor Kernfysica en Hoge Energie Fysica (NIKHEF), Science Park, Amsterdam, The Netherlands

78 Stichting Astronomisch Onderzoek in Nederland (ASTRON), Dwingeloo, The Netherlands

${ }^{79}$ Universiteit van Amsterdam, Faculty of Science, Amsterdam, The Netherlands

${ }^{80}$ Case Western Reserve University, Cleveland, OH, USA

${ }^{81}$ Colorado School of Mines, Golden, CO, USA

82 Department of Physics and Astronomy, Lehman College, City University of New York, Bronx, NY, USA

${ }^{83}$ Louisiana State University, Baton Rouge, LA, USA
${ }^{84}$ Michigan Technological University, Houghton, MI, USA

${ }^{85}$ New York University, New York, NY, USA

${ }^{86}$ Pennsylvania State University, University Park, PA, USA

87 University of Chicago, Enrico Fermi Institute, Chicago, IL, USA

${ }^{88}$ University of Delaware, Bartol Research Institute, Department of Physics and Astronomy, Newark, USA

${ }^{89}$ University of Nebraska, Lincoln, NE, USA

a School of Physics and Astronomy, University of Leeds, Leeds, United Kingdom

${ }^{b}$ Max-Planck-Institut für Radioastronomie, Bonn, Germany

${ }^{c}$ Fermi National Accelerator Laboratory, USA

$d$ also at Universidade Federal de Alfenas, Poços de Caldas, Brazil

${ }^{e}$ Colorado State University, Fort Collins, CO, USA

${ }^{f}$ now at the Hakubi Center for Advanced Research and Graduate School of Science at Kyoto University

$g$ also at Karlsruhe Institute of Technology, Karlsruhe, Germany

$h$ also at University of Bucharest, Physics Department, Bucharest, Romania

${ }^{i}$ now at European Southern Observatory, Garching bei München, Germany 